\title{
Excited-State Absorption of Uracil in the Gas Phase: Mapping the Main Decay Paths by Different Electronic Structure Methods
}

Fedotov, Daniil A; Paul, Alexander C; Posocco, Paolo; Santoro, Fabrizio; Garavelli, Marco; Koch, Henrik; Coriani, Sonia; Improta, Roberto

Published in:

Journal of Chemical Theory and Computation

Link to article, DOI:

10.1021/acs.jctc.0c01150

Publication date:

2021

Document Version

Version created as part of publication process; publisher's layout; not normally made publicly available

Link back to DTU Orbit

Citation $(A P A)$ :

Fedotov, D. A., Paul, A. C., Posocco, P., Santoro, F., Garavelli, M., Koch, H., Coriani, S., \& Improta, R. (2021). Excited-State Absorption of Uracil in the Gas Phase: Mapping the Main Decay Paths by Different Electronic Structure Methods. Journal of Chemical Theory and Computation, 17(3), 1638-1652.

https://doi.org/10.1021/acs.jctc.0c01150

\section{General rights}

Copyright and moral rights for the publications made accessible in the public portal are retained by the authors and/or other copyright owners and it is a condition of accessing publications that users recognise and abide by the legal requirements associated with these rights.

- Users may download and print one copy of any publication from the public portal for the purpose of private study or research.

- You may not further distribute the material or use it for any profit-making activity or commercial gain

- You may freely distribute the URL identifying the publication in the public portal 


\title{
Excited-State Absorption of Uracil in the Gas Phase: Mapping the Main Decay Paths by Different Electronic Structure Methods
}

\author{
Daniil A. Fedotov, Alexander C. Paul, Paolo Posocco, Fabrizio Santoro, Marco Garavelli, Henrik Koch, \\ Sonia Coriani,* and Roberto Improta*
}

Cite This: https://dx.doi.org/10.1021/acs.jctc.0c01150

Read Online

ACCESS I

Llll Metrics \& More

Article Recommendations

Supporting Information

ABSTRACT: We present a computational study of the one-photon and excited-state absorption (ESA) from the two lowest energy excited states of uracil in the gas phase: an $n \pi^{*}$ dark state $(\mathbf{1 n})$ and the lowest energy bright $\pi \pi^{*}$ state $(\mathbf{1} \pi)$. The predictions of six different linear response electronic structure methods, namely, TD-CAM-B3LYP, EOM-CCSD, EOM-CC3, $\operatorname{ADC}(2), \operatorname{ADC}(2)-x$, and $\operatorname{ADC}(3)$ are critically compared. In general, the spectral shapes predicted by TD-CAM-B3LYP, EOM-CCSD, EOM-CC3,

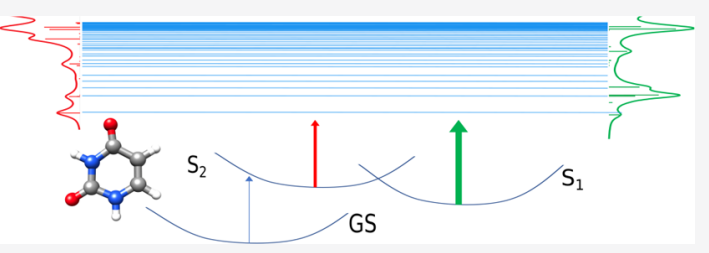
and $\mathrm{ADC}(3)$ are fairly similar, though the quality of TD-CAM-B3LYP slightly deteriorates in the high-energy region. By computing the spectra at some key structures on different potential energy surfaces (PES), that is, the Franck-Condon point, the 1n minimum, and structures representative of different regions of the $1 \boldsymbol{\pi}$ PES, we obtain important insights into the shift of the ESA spectra, following the motion of the wavepacket on the excited-state PES. Though $\mathbf{1} \boldsymbol{\pi}$ has larger ESA than 1n, some spectral regions are dominated by these latter signals. Aside from its methodological interest, we thus obtain interesting indications to interpret transient absorption spectra to disentangle the photoactivated dynamics of nucleobases.

\section{INTRODUCTION}

The development of pump-probe spectroscopy has allowed tremendous advances in our understanding of the excited-state dynamics of molecules and materials. ${ }^{1-3}$ In this field, transient absorption (TA) has surely been one of the most profitably used techniques. ${ }^{2,4}$ However, the interpretation of a TA spectrum, especially for medium/large molecular systems in the condensed phase, is seldom straightforward. It is indeed the result of different processes [ground-state (GS) bleaching, stimulated emission and excited-state absorption, hereafter ESA], not easily disentangled. ${ }^{5}$ In particular, it is not trivial to predict and interpret ESA spectra: the manifold of the excited states is often very dense and many excited states can give rise to appreciable ESA in a given spectral region. A correct assignment/interpretation of the ESA and, consequently, of TA spectra would thus enormously benefit from computation of ESA by means of quantum mechanical methods. ${ }^{6}$ However, the number of computational studies tackling this task is still relatively limited, especially when compared to the huge amount of computational data available for one-photon

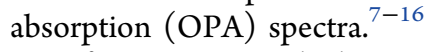

In fact, accurate calculation of ESA poses several nontrivial challenges. The computational cost is usually high since a large number of excited states has to be computed. It is also necessary to attain an accurate treatment of higher-lying excited states in a region where the density of states is large and the double excitations can play an important role.

In the high-energy region of the spectrum, the role of double and higher (triple, quadruple) excitations cannot be discarded a priori. As a consequence, several computational issues, for example, the choice of the basis set or the selection of the active space in a multiconfigurational calculation, can be more delicate than in standard excited-state calculations. More importantly, there are several key methodological aspects to be considered.

Methods to obtain ESA can be broadly divided in two groups: (1) state-specific approaches, where initial and final state wave functions are explicitly optimized and transition moments between them are evaluated and (2) response/ propagator methods, where the excited-state response is obtained directly from the GS wave function, without any knowledge of the excited-state wave functions. Intermediate between the two groups are response variants where a linear response approach (like in OPA) is applied on a specific representation of the chosen initial excited state, obtained, for example, by applying some overlap criterion like in the maximum overlap method (MOM) ${ }^{17}$ or the square-minimization $\operatorname{method}^{18}$ to avoid variational collapse during the nonaufbau self-consistent-field optimization of the excited state. The "black-box" nature of response/propagator methods makes them particularly attractive to use. However, since the

Received: November 2, 2020 
spectra are computed as transitions between excited states generated from the GS, it remains unclear, and it is partly addressed in this study, whether a chosen electronic structure method is capable of capturing all spectral signatures, and in particular those corresponding to excitation channels with double or higher excitation character. A similar problem has recently been discussed in connection with simulations of excited-state X-ray absorption. ${ }^{19-21}$

A critical comparison between different methods can provide useful benchmarks to understand advantages and limitations of different approaches. In this spirit, we here report a study of the ESA for a prototypical molecule, uracil, tackled by six different response methods, namely, equation-of-motion coupled cluster singles and doubles (EOM-CCSD), ${ }^{22-24}$ EOM coupled cluster singles, doubles and approximate triples (EOM-CC3), ${ }^{25}$ time-dependent density functional theory (TD-DFT) in combination with the CAM-B3LYP functional, and algebraic diagrammatic construction to second and third order, $\operatorname{ADC}(2), \operatorname{ADC}(2)-x$, and $\operatorname{ADC}(3) .{ }^{26}$ We focus on the two lowest energy excited states: a dark state with $\mathrm{n} \pi^{*}$ character $\left(S_{1}\right.$ in the Franck-Condon (FC) point, hereafter 1n) and a bright $\pi \pi^{*}$ state $\left(S_{2}\right.$ in the FC region, hereafter $\left.1 \pi\right)$. The ESA will be computed for different representative structures of the potential energy surface (PES): the GS minimum (FC point), the minimum of $\mathbf{1 n}$ (plus a planar pseudo-minimum), three points of the $1 \pi$ PES, that is, a planar pseudo-minimum and two representative structures of the path connecting the FC point to the lowest energy conical intersection (CI) with the GS. In this way, we should obtain a more complete picture than the one obtained by looking only at the FC point, as often carried out in many comparative studies, since for planar structures, as it happens for uracil, many electronic transitions are decoupled by symmetry. At the same time, we can get very useful information to interpret the TA spectra of uracil, which has been studied in the past in order to understand the photoactivated dynamics of this molecule. Actually, the possible role of dark states in the excited-state decay of pyrimidines after UV absorption is a lively debated topic, ${ }^{27-32}$ an exhaustive review falling outside the aims of this paper, for its possible implication in the photophysics of nucleic acids. In this respect, we hope to assess whether characteristic ESA signals for $1 \mathrm{n}$ and $\mathbf{1} \boldsymbol{\pi}$ exist and can be used to discriminate between the two and to obtain indications on how the states change along the photoexcited path, laying the groundwork for future studies in the condensed phase. From the methodological point of view, it is worth to notice that since the OPA of uracil, like for the other nucleobases, is peaked at $\sim 250-270$ $\mathrm{nm}$, the final states of ESA lie at high energies so that their description is particularly challenging for electronic structure methods. As our study shows, it is comforting that, overall, the methods examined provide a consistent picture of the OPA and of the ESA of $1 n$ and $1 \boldsymbol{\pi}$ of uracil. On the other hand, some differences appear; the spectral shapes obtained with TD-CAM-B3LYP, EOM-CCSD, EOM-CC3, and ADC(3) are indeed rather similar, whereas $\mathrm{ADC}(2)$ and, especially, $\mathrm{ADC}(2)-\mathrm{x}$ spectra exhibit some different features.

\section{COMPUTATIONAL DETAILS}

Geometry optimizations were performed at the MP2/cc-pVTZ level for the GS and at the EOM-CCSD/aug-cc-pVDZ level for the excited states. The molecular Hessian was also calculated and, unless otherwise specified, no imaginary frequencies were obtained.
The OPA and ESA calculations in the gas phase were performed using EOM-CCSD, EOM-CC3, and TD-DFT, with the CAM-B3LYP functional, $\operatorname{ADC}(2), \operatorname{ADC}(2)-x$, and $\mathrm{ADC}(3)$. Dunning's correlation consistent basis set aug-ccpVDZ was adopted in all calculations. ${ }^{33}$ Test calculations were also carried out at the EOM-CCSD level using the aug-ccpVDZ basis set incremented with Rydberg-type basis functions, chosen according to the prescription of Kaufmann, Baumeister, and Jungen (KBJ), ${ }^{34}$ with quantum numbers $n=3$, 3.5, and 4. The EOM-CCSD spectrum obtained with the augcc-pVDZ + KBJ basis is reported in the Supporting Information.

The ESA computed at the FC point at the RASPT2 level, as implemented in the MOLCAS 8 package, ${ }^{35}$ have been recently published for all canonical nucleobases in the gas phase, including uracil. ${ }^{15}$ These data have also been used in this work as additional benchmark for $1 \boldsymbol{\pi}$ ESA.

The RASPT2 computations are based on an adapted (ANO)-type triple- $\zeta$ basis set with removed Rydberg-like orbitals and a RAS $(0,0-10,8-2,12)$ active space including all $\pi$ and $\pi^{*}$ valence orbitals in a complete active space (i.e., with full configuration interaction) plus additional $\pi^{*}$ orbitals (12) with up to two electron permutations allowed in order to further increase the dynamic correlation toward convergence (see ref 15 for details). Thirty singlet excited states were included in the multiconfigurational state-average treatment in order to account for enough states to monitor all ESA signals of interest.

The spectra were computed for the GS minimum (FC geometry) and for a few representative points of the PES of 1 n and $1 \pi$. In detail, for $1 \mathbf{n}$, we considered two structures: the absolute minimum (optimized starting from the minimum reported by Matsika ${ }^{36}$ that was obtained at the multireference configuration interaction MRCI1/cc-pVDZ level) and a stationary point (identified by the label $\mathrm{pl}$ ), optimized under the constraint of planarity. This planar stationary point is less stable than the absolute minimum by only $0.11 \mathrm{kcal} / \mathrm{mol}$ and exhibits an imaginary frequency of $157.9 \mathrm{~cm}^{-1}$.

In fact, the minimum of 1 n (coordinates given in the Supporting Information) is also almost planar, and its geometry is very similar to the planar stationary point but for a very small out of plane distortion of $\mathrm{H} 3$ (the $\mathrm{H}$ atom bonded to N3). We also considered two non-planar structures of $1 \mathrm{n}$, obtained at the M052X/aug-cc-pVDZ and M052X/6$31+\mathrm{G}(\mathrm{d}, \mathrm{p})$ levels of theory, labelled R4 and R5. The Cartesian coordinates (and the ESA spectra) for these two geometries can be found in the Supporting Information.

For $1 \boldsymbol{\pi}$, we also carried out a constrained planar minimization, and the resulting structure exhibits two imaginary frequencies (465.33 and $\left.200.66 \mathrm{~cm}^{-1}\right)$. Our attempts of optimizing an absolute minimum for $\mathbf{1} \boldsymbol{\pi}$ were unsuccessful. As discussed below, a barrierless path on $\mathbf{1} \pi$ connects the FC point with a CI with the GS. We therefore selected three additional non-planar (npl) structures (labeled as npl1, npl2, and npl3) along this decay path, as predicted by CAM-B3LYP via different levels of calculation, namely CAMB3LYP/aug-cc-pVTZ, CAM-B3LYP/6-31+G(d,p), and PCM/ CAM-B3LYP/6-31+G(d,p). These structures (whose energy gradient is very small, see the Supporting Information) are rather similar, with the pyrimidine ring bent, and the $\mathrm{C} 5$ and C6 atoms slightly pyramidalized. In the main text, we shall report the spectra obtained for the more distorted one (npl3), 


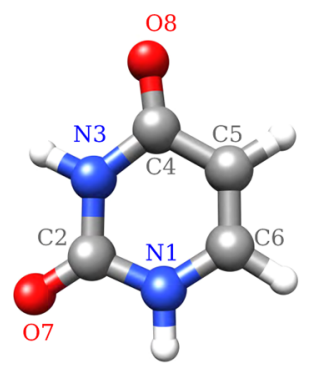

a)

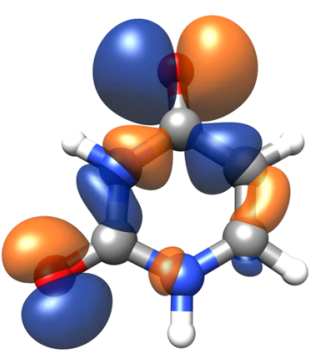

b)

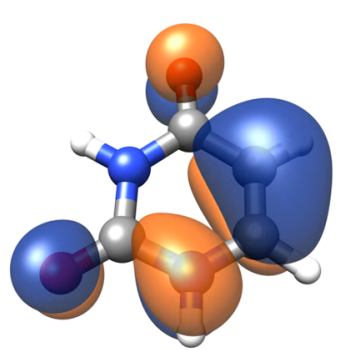

c)

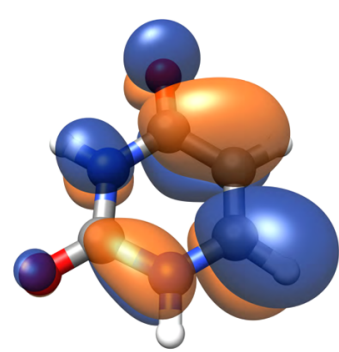

d)

Figure 1. (a) Uracil, including atom labeling. Schematic drawing of the frontier orbitals, computed at the CAM-B3LYP/aug-cc-pVDZ level, involved in the lowest energy transitions of uracil in the gas phase: (b) HOMO - 1; (c) HOMO; (d) LUMO.

whereas npl1 and npl2 spectra can be found in the Supporting Information.

The schematic drawing of the planar stationary points and the distorted npl3 structure are shown in Figure 2.

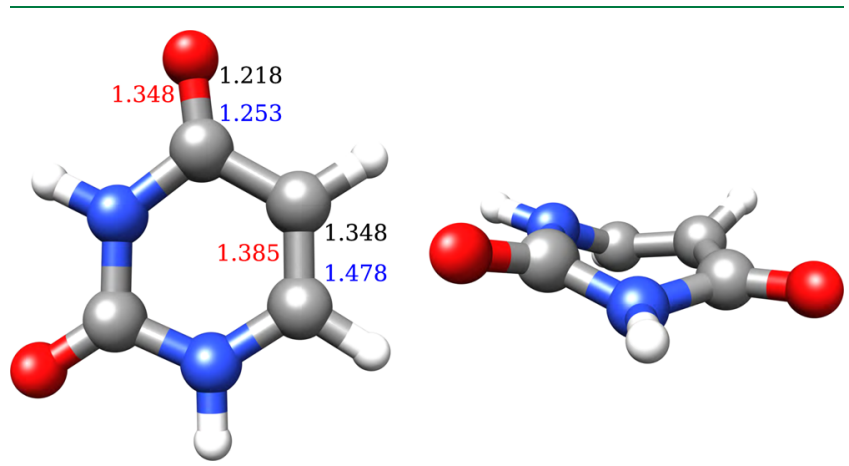

Figure 2. Optimized planar structures with representative bond lengths (in $\AA$ : GS: black; 1n: red; $\mathbf{1} \boldsymbol{\pi}$ : blue) and the distorted npl3 structure.
The spectra computed on the planar (pseudo-)minima of the GS, the $1 n$, and the $1 \boldsymbol{\pi}$ state optimized at the CAMB3LYP/aug-cc-pVDZ level are also reported in Supporting Information (see Figures S2-S4).

All MP2, CCSD, and CC3 calculations were performed using the frozen core approximation. The MP2 GS optimization was performed with Gaussian $16,{ }^{37}$ whereas the excited-state optimizations were carried out with Q-Chem 5.0. ${ }^{38}$ Hessian calculations were carried out with CFOUR. ${ }^{39}$

The OPA and ESA spectral calculations at the EOM-CCSD and $\mathrm{ADC}$ levels were performed using Q-Chem 5.0, ${ }^{38}$ whereas the OPA and ESA spectra at the CAM-B3LYP level were obtained using Dalton. ${ }^{40}$ The CC3 calculations were carried out using the eT code. ${ }^{41}$

Spectra are reported as decadic molar extinction coefficients $\epsilon(\omega)$ as a function of the frequency

$$
\epsilon(\omega)=C \omega \sum_{j} g\left(\omega, \omega_{j}\right) \frac{3 f_{j}}{2 \omega_{j}}
$$
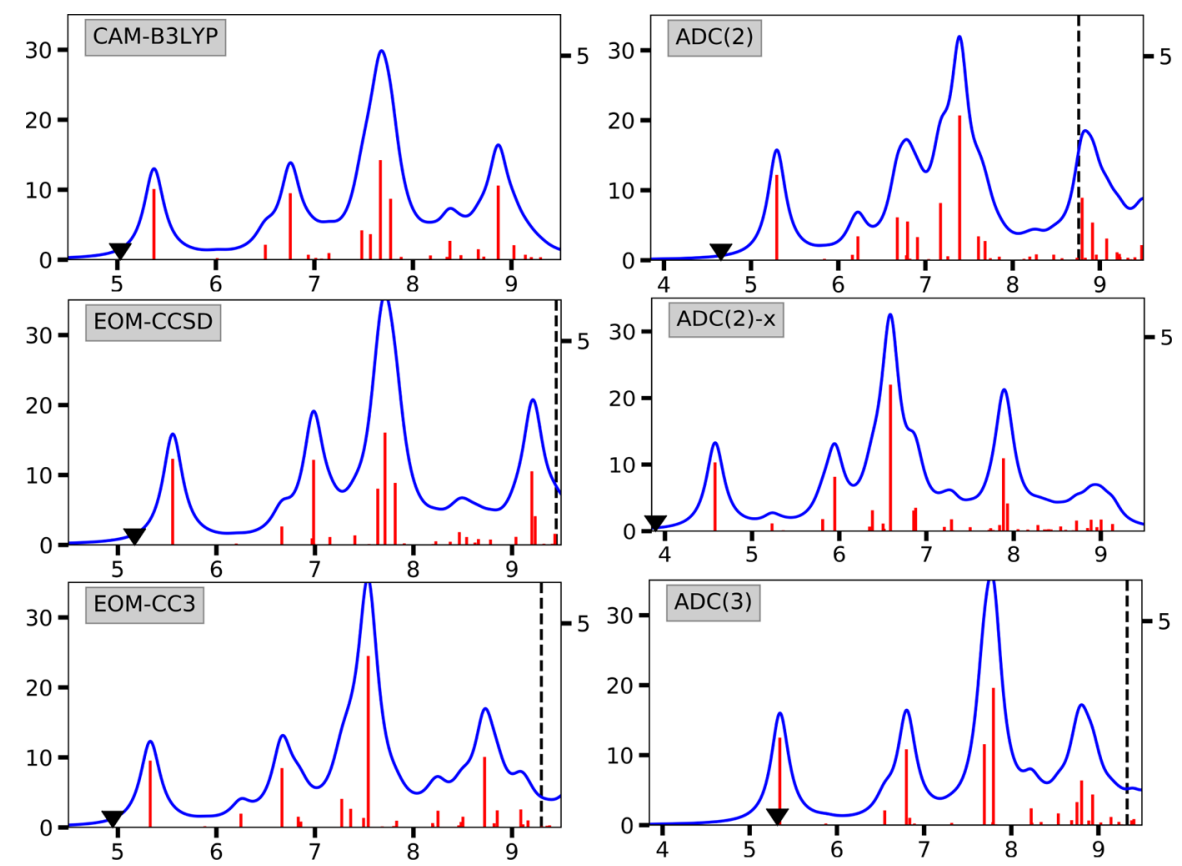

Figure 3. Uracil. OPA spectra at the (MP2 optimized) FC geometry for all six electronic structure methods. A black triangle marks the position of the dark $1 n$ state. A vertical dashed line corresponds to the first IE. The first IE of CAM-B3LYP and ADC(2)-x is outside the shown frequency ranges. In abscissa, we report the excitation energy in electron Volts and in left ordinate, the decadic molar extinction coefficient, ${ }^{42} \epsilon(\omega) \times 10^{-3}$, in units $\mathrm{M}^{-1} \mathrm{~cm}^{-1}$. On the right ordinate is the oscillator strength, $f \times 10$. 
where $\omega_{j}$ and $f_{j}$ are the excitation energies and oscillator strengths of the stick spectra, respectively, $\omega$ is the laser frequency, and $g\left(\omega, \omega_{j}\right)$ is the Lorentzian broadening function

$$
g\left(\omega, \omega_{j}\right)=\frac{\gamma}{\pi\left[\left(\omega-\omega_{j}\right)^{2}+\gamma^{2}\right]}
$$

with $\gamma=0.0045563$ Hartree $\left(1000 \mathrm{~cm}^{-1}\right)$ as half width at halfmaximum. The prefactor $C=703.301$ yields the decadic molar extinction coefficient in units of $\mathrm{L} \mathrm{mol}^{-1} \mathrm{~cm}^{-1}$ when excitation energies and frequencies are given in Hartree (see ref 42 for details).

For all methods, we have computed the first ionization energy (IE) as follows

- For EOM-CCSD, by standard EOM-IP-CCSD calculations;

- For EOM-CC3, as excitation into a super-diffuse orbital; $^{43-45}$

- For $\mathrm{ADC}(2)$ and $\mathrm{ADC}(3)$, by standard $\mathrm{ADC}(2)$-IP and $\mathrm{ADC}(3)-\mathrm{IP}$ calculations;

- For CAM-B3LYP, both by Koopmans' theorem, IE = $-\epsilon_{\mathrm{HOMO}}$, and directly as difference between the total energy of the cation and the total energy of the neutral at the FC geometry.

- The difference of total energy between the cation and the GS was used as estimate of the IP for $\operatorname{ADC}(2)$ - $x$

Estimates of the first ionization limit in the ESA spectra were obtained as difference between the first IE of the GS and the excitation energy of the valence excited state considered.

The first ionization thresholds are indicated in the figures by vertical dashed lines. All values are collected in Tables S3 and S4 in Supporting Information. Estimates of the IE have been included to pinpoint that ESA predictions relative to final states that fall above the first IE-if not even transitions to high-energy states close to the ionization limit-must be handled with some caution. Above the ionization limit, continuum states are present, which are badly described in square-integrable basis sets. Some of the "final" states may therefore correspond to unphysical representations of the continuum. $^{46,47}$ For TD-DFT, as discussed elsewhere, ${ }^{48-50}$ when approaching the ionization threshold of the highest occupied molecular orbital (HOMO) Kohn-Sham orbital, spurious mixing with ionic states may arise. ${ }^{48-50}$

\section{RESULTS}

3.1. One-Photon Absorption Spectra. In Figure 3, we report the OPA spectra computed with six methods at the FC geometry. The raw data for the figure are tabulated in Tables S5, S7, and S9-S12. Tables S20-S24 contain the natural transition orbitals (NTOs) of the main OPA transitions for all methods except EOM-CC3.

Our spectra are generally consistent with those obtained in several computational studies of uracil. ${ }^{28,51,52}$ EOM-CCSD, CAM-B3LYP, $\mathrm{ADC}(3)$, and EOM-CC3 provide quite similar OPA spectra at the FC geometry, with four intense bands: at $\sim 5.2 \mathrm{eV}$, below $7 \mathrm{eV}$, at 7.5-7.7 eV, and just below $9 \mathrm{eV}$. On an average, the EOM-CCSD peaks are slightly blue-shifted by 0.1-0.2 eV with respect to the other three methods. The computed relative intensities are also similar, with the third band being the strongest, while the other three have comparable intensities. The spectral patterns predicted by $\operatorname{ADC}(2)-x$ and $\operatorname{ADC}(2)$ are overall consistent with the picture just described, with two noticeable differences: $\operatorname{ADC}(2)-x$ peaks are significantly red-shifted (by $\sim 1 \mathrm{eV}$ ) with respect to the other methods reported in Figure 3 and, in fact, the most intense transitions fall at $\sim 4.6, \sim 6, \sim 6.6$, and $\sim 8 \mathrm{eV}$, respectively. The energy gap between the second and the third peak also decreases. This latter feature is even more prominent in the $\operatorname{ADC}(2)$ spectrum. $\operatorname{ADC}(2)$ yields several transitions of similar intensity between 6 and $7 \mathrm{eV}$, resulting in a less resolved spectrum in that region.

Finally, as shown in the Supporting Information, the OPA spectra computed at the EOM-CCSD and CAM-B3LYP level at the minimum optimized at the CAM-B3LYP level are almost coincident with those shown in Figure 3.

The experimental vapor phase spectrum of uracil ${ }^{53}$ is available up to $7 \mathrm{eV}$. With the exception of the $\mathrm{ADC}(2)-\mathrm{x}$ one (which is red-shifted), the computed spectra are fully consistent with the experimental one, which shows a broad band peaking at $5.17 \mathrm{eV}$, with a shoulder at $\sim 6.05$, and another peak at $6.63 \mathrm{eV}$. As also shown by previous studies on cytosine, ${ }^{54,55}$ vibrational effects ${ }^{56,57}$ can give account of the small blue-shift of the excitation energy to $1 \pi$ and the position of the first experimental maximum.

For what concerns the assignment of the spectra, all methods considered agree that the lowest energy bright transition can be described as a HOMO $\rightarrow$ LUMO excitation (the $1 \pi$ state), with antibonding character with respect to the C5-C6 double bond (see Figure 1 and Tables S1 in Supporting Information).

The second intense peak also has $\pi \pi^{*}$ character, that is, from the HOMO to the second lowest energy $\pi^{*} \operatorname{MO}(3 \pi)$, according to all methods. It is preceded by a weak one, involving the second highest energy $\pi$ orbital and the lowest unoccupied molecular orbital (LUMO) $(2 \pi)$. As anticipated above, $\operatorname{ADC}(2)$ provides a slightly different picture of the spectral region at $6-7 \mathrm{eV}$ : the $3 \mathrm{~A}^{\prime}$ state (second band) has a predominant Rydberg component (see NTO in the Supporting Information) and the third band involves, beside the $3 \boldsymbol{\pi}$ state, two other transitions of intensity comparable to it but with dominant $\mathrm{n} \rightarrow$ Rydberg character.

The transitions responsible for the band peaking at 7.5-7.8 $\mathrm{eV}$ derive from the mixing of a $\pi \pi^{*}$ excitation (from the second highest energy $\pi$ to the second lowest energy $\pi^{*}$ orbital, $4 \pi$ ) with several $\mathrm{n} \rightarrow$ Rydberg states. However, according to $\mathrm{ADC}(3)$, the most intense transition has prevalent $\mathrm{n} \rightarrow$ Rydberg character, but, also in this case, it is strongly mixed with $4 \pi \cdot \operatorname{ADC}(2)$ - $x$ also assigns the third intense peak (falling at $\sim 6.5 \mathrm{eV}$ ) to the transition to $4 \pi$. Probably, the mixing with $\mathrm{n} \rightarrow$ Rydberg states explains why, although the broadened OPA spectrum provided by the different methods in that region is rather similar, the intensities on the individual transitions are different. For example, in the EOM-CC3 spectrum, we have a single transition, at $\sim 7.5 \mathrm{eV}$, much more intense than the other ones, whereas, according to CAMB3LYP and EOM-CCSD, the different transitions contributing to this band have a similar intensity. On the other hand, the CAM-B3LYP spectrum computed at the CAM-B3LYP minimum (see Figure S2) provides an intensity pattern much more similar to the EOM-CC3 one, suggesting that vibronic interactions can modulate the coupling between bright and dark states and these differences should not be overinterpreted.

Finally, the intense transition at $\sim 8.9 \mathrm{eV}$ [at $\sim 7.9$ according to $\mathrm{ADC}(2)-\mathrm{x}]$ is also due to a $\pi \pi^{*}$ (from the third highest 

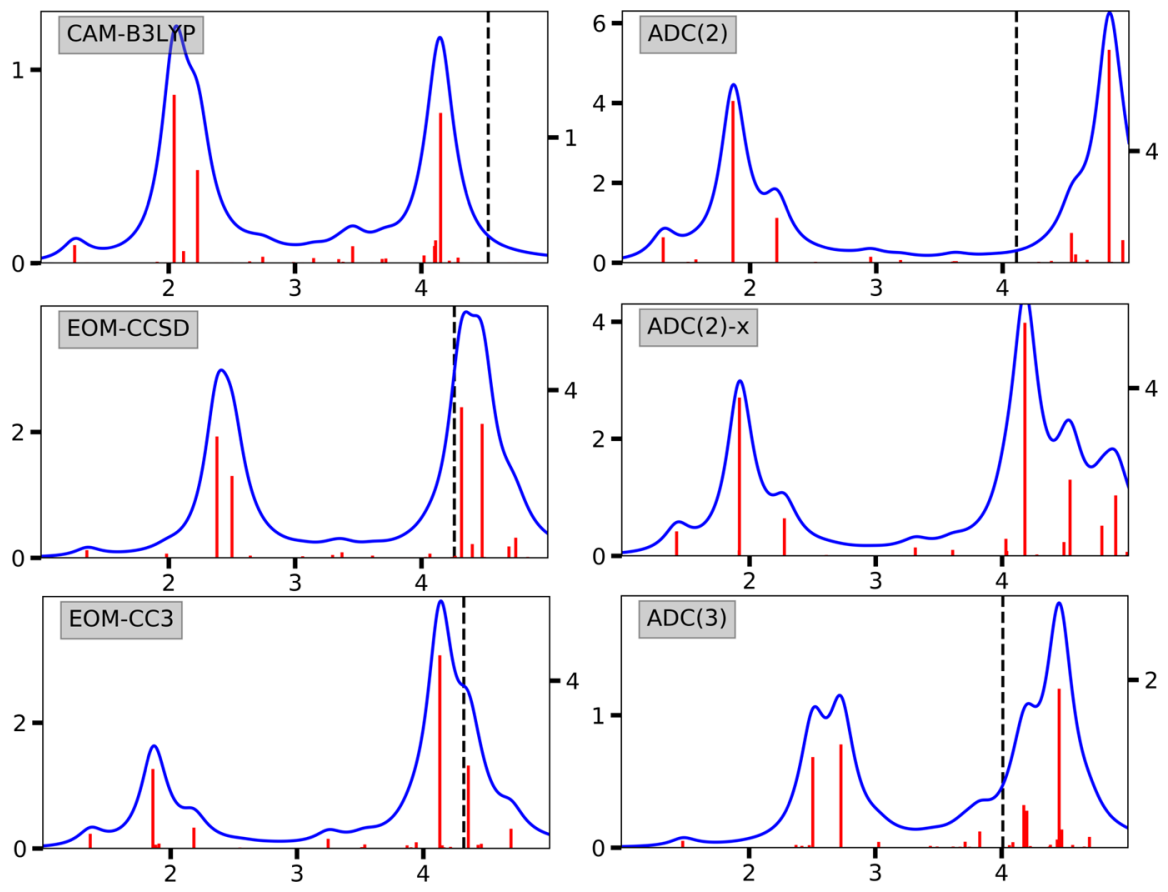

Figure 4. Uracil. ESA spectra of $\mathbf{1 n}$ at the (MP2 optimized) FC geometry for six electronic structure methods. A vertical dashed line indicates the estimated value of the first IE in the excited state, obtained as IE of the GS minus the energy of $1 \mathbf{n}$ (see text for details). In abscissa, we report the excitation energy in electron Volts and in left ordinate, the decadic molar extinction coefficient, ${ }^{42} \epsilon(\omega) \times 10^{-3}$, in units $\mathrm{M}^{-1} \mathrm{~cm}^{-1}$. On the right ordinate is the oscillator strength, $f \times 10^{1}$. Note that the ordinate of each panel has a different scale. A figure using the same scale is reported in Supporting Information (see Figure S8).

energy $\pi$ to the $\mathrm{LUMO}, 5 \pi)$ though, according to $\operatorname{ADC}(3)$, the most intense peak has $\mathrm{n} \rightarrow$ Rydberg character.

The involvement of states of Rydberg character raises the question on whether the aug-cc-pVDZ basis is sufficiently flexible to describe such transitions. We have verified this by computing the OPA spectrum at the EOM-CCSD level with the extended aug-cc-pVDZ + KBJ basis set. The resulting spectrum, as shown in Figure S1, confirms that the aug-ccpVDZ basis is sufficiently flexible.

Since they could be active in the ESA spectra, it is also important to analyze the weakly allowed transitions, like those with $\mathrm{n} \pi^{*}$ character, not affecting the OPA spectra. Independent of the adopted method, the lowest energy excited state in the $\mathrm{FC}$ region corresponds to a $\mathrm{n} \pi^{*}$ transition (hereafter 1n), which can be described as an excitation from the lone pair (LP) of the carbonyl oxygen O8 toward the $\pi^{*}$ LUMO (see Figure 1 and the characterization via the NTOs in Table S2).

The energy gap between $\mathbf{1} \boldsymbol{\pi}$ and $\mathbf{1 n}$ significantly changes with the level of theory. It is $\sim 0.35-0.39 \mathrm{eV}$, according to EOM-CCSD, EOM-CC3, and CAM-B3LYP; $\sim 0.65 \mathrm{eV}$, according to $\operatorname{ADC}(2)$ and $\operatorname{ADC}(2)-x$; and only $0.03 \mathrm{eV}$, according to $\operatorname{ADC}(3)$. The second $\mathrm{n} \pi^{*}$ transition (hereafter 2n) involves excitation from the LP of carbonyl $\mathrm{O} 7$ atom toward the second lowest energy $\pi^{*}$ orbital, as it can be seen from the NTOs in Figures S5, S7, and S9-S12).

Its relative stability with respect to 1 n does not significantly change with the method, being in the range $1.3 \mathrm{eV}$ [according to EOM-CCSD, EOM-CC3, $\mathrm{ADC}(2)$, and CAM-B3LYP] to $\sim 1.6 \mathrm{eV}$ [according to $\mathrm{ADC}(3)]$. As a consequence, its position with respect to the bright transitions exhibit the same trends as described for $\mathbf{1 n}$.
A detailed assignment of the OPA can be found in the Supporting Information. In the next section, we will analyze the transitions that are more important for the assignment of the ESA.

Summarizing the results of this section, the methods examined provide similar OPA spectra, with four main bands clearly recognizable, with the partial exception of $\mathrm{ADC}(2)$, where the spectral region between the second and the third band is much more congested.

Finally, we assess the importance of double excitations on the electronic transitions identified by OPA. To this end, we consider the relative importance of single excitations $\left(\% R_{1}\right)$ in the excitation vectors for CCSD and CC3 (see Table S13 in Supporting Information). While the percentage weight of single excitations of the transitions is slightly reduced in CC3 compared to CCSD, all transitions remain dominated by single excitations (\% $R_{1}>91$ in all cases). Nonetheless, moving toward higher energies, the improved description of the excited states from $\mathrm{CC} 3$ results in some states moving up and down in energy and in some intensity redistribution among the individual states.

3.2. Excited-State Absorption. 3.2.1. Franck-Condon Point. We start our analysis of the ESA from the $1 \mathrm{n}$ and $\mathbf{1} \pi$ states at the FC point. In addition to providing useful methodological information, ESA from the FC region can affect the appearance of the experimental ESA (or TA) spectra. Moreover, since the $\mathbf{1} \boldsymbol{\pi}$ spectroscopic state decays on a $\leq 1$ ps time-scale $^{28,58}$ (and this is also the limit for the possible population of $\mathbf{1 n}$ ), ESA from the FC region could provide a significant contribution to the total signal.

As shown in Figure 4, the ESA spectra below $4 \mathrm{eV}$ computed for $1 \mathrm{n}$ by the different methods are rather similar. We find a first, relatively weak transition at $\sim 1.3 \mathrm{eV}$, associated to the 1 n 

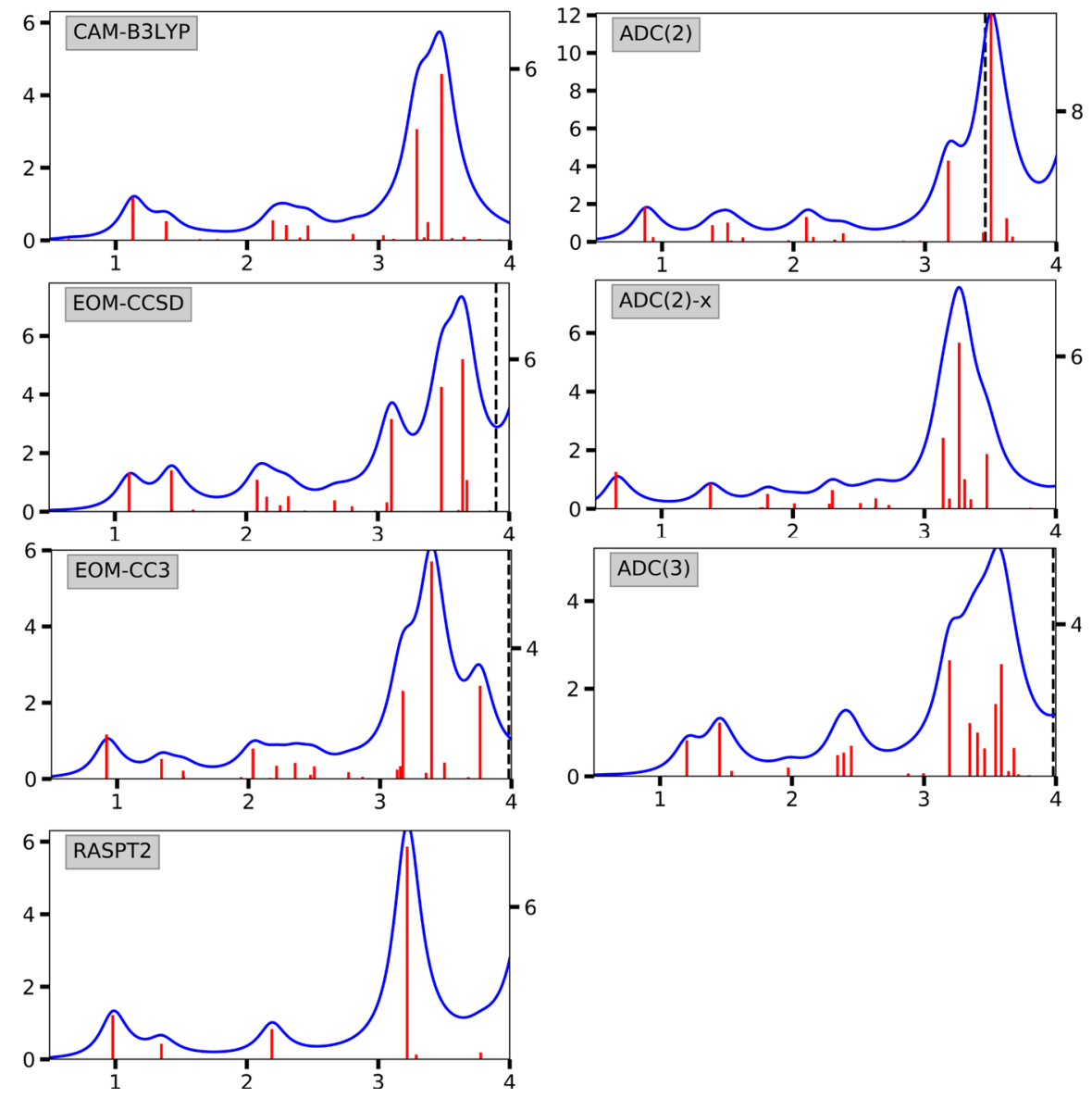

Figure 5. Uracil. ESA spectra of $1 \boldsymbol{\pi}$ at the (MP2 optimized) FC geometry for all seven electronic structure methods. A vertical dashed line indicates the position of the first IE estimated, as described in the computational details. The estimated IE of ADC(2)-x is outside the plotted frequency range $(5.44 \mathrm{eV})$. That of RASPT2 was not determined. In abscissa, we report the excitation energy in electron Volts and in left ordinate, the decadic molar extinction coefficient, ${ }^{42} \epsilon(\omega) \times 10^{-3}$, in units $\mathrm{M}^{-1} \mathrm{~cm}^{-1}$. On the right ordinate is the oscillator strength, $f \times 10^{2}$.

$\rightarrow 2 \mathrm{n}$ transition, before two stronger peaks at $2.0-2.5 \mathrm{eV}$. In detail, EOM-CCSD predicts a weak feature at $1.35 \mathrm{eV}$, a weaker one at $2 \mathrm{eV}$, followed by two strong peaks at $2.4-2.5$ $\mathrm{eV}$. These two latter are due to the transitions to $7 \mathrm{~A}^{\prime \prime}$ and $8 \mathrm{~A}^{\prime \prime}$ excited states, which mainly involve the excitation from the LP of $\mathrm{O} 8$ and $\mathrm{O} 7$, respectively, to the second lowest energy $\pi^{*}$ orbital, as shown by their NTOs in Tables S20 in Supporting Information. In the CAM-B3LYP spectra, the two peaks are $\sim 0.2 \mathrm{eV}$ red-shifted and also involve the excitation from the combination of the LPs of the two oxygen atoms to the two lowest energy $\pi^{*}$ states. Also according to CAM-B3LYP, these pairs of intense transitions are accompanied by two very weak features at $\sim 1.3$ and $\sim 2.1 \mathrm{eV}$. The EOM-CC3 spectrum below $4 \mathrm{eV}$ is similar to the CAM-B3LYP one, apart from a moderate red-shift $(\sim 0.1 \mathrm{eV})$ of the two peaks at $\sim 2 \mathrm{eV}$.

According to $\mathrm{ADC}(2)$, the intense peak just below $2 \mathrm{eV}$ is assigned to the transition to a state involving the excitation from the O7 LP toward the LUMO, whereas the one at $\sim 2.2$ $\mathrm{eV}$ involves an excited state that can be described as an excitation from a combination of the O7 and O8 LP to the second lowest energy $\pi^{*}$ state. The assignment of the $\operatorname{ADC}(2)-x$ and the $\mathrm{ADC}(3)$ spectra is similar to that of CAM-B3LYP.

Shortly, the six methods provide a similar spectral pattern (with a fairly consistent assignment) of this low-energy region part of the ESA spectra of $\mathbf{1 n}$. On the other hand, remarkable differences in the computed intensities are found as CAM-
B3LYP, EOM-CC3, and ADC(3) intensities are roughly onehalf of those predicted by the other three methods.

All methods predict a relatively intense band above $4 \mathrm{eV}$, whose intensity depends however on the adopted method. CAM-B3LYP predicts that this high-energy band is slightly less intense than the one falling at $\sim 2.1 \mathrm{eV}$, whereas at the other extreme, $\mathrm{ADC}(3)$ and $\mathrm{EOM}-\mathrm{CC} 3$ predict that this latter one is roughly half as intense. In detail, CAM-B3LYP predicts a "cluster" of peaks in the $4-4.5 \mathrm{eV}$ range. However, only one bears a significant oscillator strength at $4.15 \mathrm{eV}$, and it involves excitation from a low-lying $\mathrm{LP} / \sigma$ orbital toward the LUMO. The other methods provide at least two intense transitions in this region. For example, in the EOM-CCSD spectrum, there are two intense transitions at $\sim 4.3$ and $\sim 4.5 \mathrm{eV}$, assigned to excitations to $22 \mathrm{~A}^{\prime \prime}$ and $24 \mathrm{~A}^{\prime \prime}$ states. The former excited state corresponds to the transition from the O8 LP to a Rydberg state, the latter corresponds to the lowest energy O8 LP to the LUMO. There are also two fairly intense transitions above 4.5 $\mathrm{eV}$.

According to $\mathrm{ADC}(2)$-x, the ESA to $17 \mathrm{~A}^{\prime \prime}$ (more intense, at $4.18 \mathrm{eV}$ ), 20A $\mathrm{A}^{\prime \prime}$ (at $4.49 \mathrm{eV}$ ), $21 \mathrm{~A}^{\prime \prime}$ (at $4.54 \mathrm{eV}$ ), and $25 \mathrm{~A}^{\prime \prime}$ and $26 \mathrm{~A}^{\prime \prime}$ states are intense. They all involve excitation from the $\mathrm{O} 8 \mathrm{LP}$ to Rydberg states. State $17 \mathrm{~A}^{\prime \prime}$ also has a minor contribution from the LP on O7, whereas states $20 \mathrm{~A}^{\prime \prime}$ and $21 \mathrm{~A}^{\prime \prime}$ also involve a $\sigma_{\mathrm{CO}} \rightarrow \pi^{*}$ contribution.

Above $4 \mathrm{eV}$, the strongest transitions at the $\operatorname{ADC}(2)$ level correspond to the excitations to $23 \mathrm{~A}^{\prime \prime}[4.54 \mathrm{eV}], 27 \mathrm{~A}^{\prime \prime}[4.84$ 

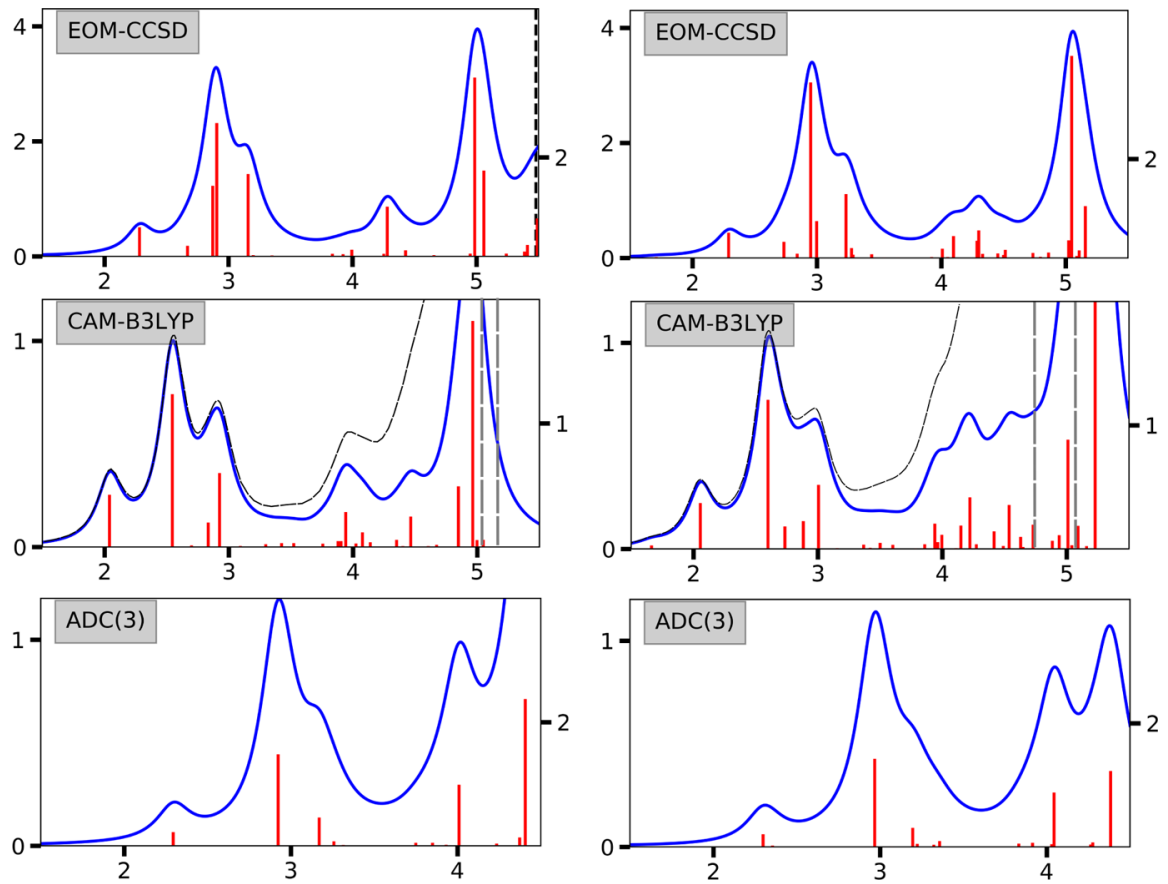

Figure 6. Uracil. ESA spectra from $1 \mathrm{n}$ at the $1 \mathrm{n}$ planar (left panels) and non-planar (right panels) EOM-CCSD stationary points. A vertical dashed line, if present, corresponds to the estimated first ionization. In abscissa, we report the excitation energy in electron Volts and in left ordinate, the decadic molar extinction coefficient, ${ }^{42} \epsilon(\omega) \times 10^{-3}$, in units $\mathrm{M}^{-1} \mathrm{~cm}^{-1}$. On the right ordinate is the oscillator strength, $f \times 10^{2}$. The gray spectral profiles and sticks in the CAM-B3LYP plots correspond to the case where artefacts due to resonances in the response equations are probably present.

$\mathrm{eV}]$, and $28 \mathrm{~A}^{\prime \prime}[4.95 \mathrm{eV}]$ states. The first state is dominated by the $\sigma_{\mathrm{CO}} \rightarrow \pi^{*}$ contribution. The most intense one, for the transition to $27 \mathrm{~A}^{\prime \prime}$, has a major contribution from the O8 LP to a $\pi^{*}$ orbital, whereas the third one is primarily a LP O7, O8 $\rightarrow$ Rydberg excited state.

The relatively more intense peaks according to $\mathrm{ADC}(3)$ originate from transitions from 1 n to $21 \mathrm{~A}^{\prime \prime}$ [4.18 eV, $f=0.51$, $22 \mathrm{~A}^{\prime \prime}[4.20 \mathrm{eV}, f=0.44]$, and $26 \mathrm{~A}^{\prime \prime}[4.46 \mathrm{eV}, f=1.89]$. These states are all of rather "mixed" character.

Finally, EOM-CC3 yields three relatively intense peaks (of progressively decreasing intensity) at $4.13,4.36$, and $4.70 \mathrm{eV}$, due to transitions to states $12 \mathrm{~A}^{\prime \prime}, 23 \mathrm{~A}^{\prime \prime}$, and $27 \mathrm{~A}^{\prime \prime}$, respectively. The two most intense peaks in CC3 above $4 \mathrm{eV}$ correspond to the two most intense peaks in CCSD above 4 $\mathrm{eV}$.

In summary, though the details and the intensities can differ, two main 1n ESA bands are predicted by all methods in the FC point: one peaking at $\sim 2 \mathrm{eV}$ [at $\sim 2.5 \mathrm{eV}$ according $\mathrm{ADC}(3)]$, the other just above $4 \mathrm{eV}$.

The ESA spectrum computed for $\mathbf{1} \boldsymbol{\pi}$ is much richer (see Figure 5), with many peaks below $4 \mathrm{eV}$.

In the low-energy region, that is below $2 \mathrm{eV}$, we can clearly distinguish the picture provided by EOM-CCSD, CAMB3LYP, and $A D C(3)$ from that of $A D C(2)$ and $\operatorname{ADC}(2)$-x. According to the first three methods, below $2 \mathrm{eV}$, there are only two fairly intense ESA features peaking at $\sim 1.1 \mathrm{eV}$ (just below $1.0 \mathrm{eV}$ for $\mathrm{CC} 3$ ) and $\sim 1.4 \mathrm{eV}$, corresponding to the transitions from $1 \pi$ to the two other lowest energy $\pi \pi^{*}$ states $2 \pi$ and $3 \boldsymbol{\pi}$ (see their characterizing NTOs in the Supporting Information). Not surprisingly, considering the differences found in the OPA spectra, $\operatorname{ADC}(2)$ and $\operatorname{ADC}(2)$-x predict a larger number of bright transitions, with intense peaks well below $1 \mathrm{eV}$. However, also for these two latter methods, the first two intense peaks in this low-energy region are assigned to the $1 \pi \rightarrow 2 \pi$ and $1 \pi \rightarrow 3 \pi$ transitions. The additional fairly intense peak predicted by $\operatorname{ADC}(2)$ at $\sim 1.5 \mathrm{eV}$ is assigned to the transition to a $\mathrm{n} \rightarrow$ Rydberg state. In the $\operatorname{ADC}(2)$-x case, this transition moves to $1.8 \mathrm{eV}$.

The EOM-CC3 spectrum exhibits a spectral pattern similar to the one of CAM-B3LYP and EOM-CCSD, but the first peak falls below $1 \mathrm{eV}$.

The ESA spectra computed at EOM-CCSD, EOM-CC3, CAM-B3LYP, and $\mathrm{ADC}(3)$ level are also fairly similar in the $2-3 \mathrm{eV}$ energy region, with four features below $2.5 \mathrm{eV}$, though the former method predicts, on average, larger intensities. Overall, all methods yield a rather shallow band covering that region, except for the $\mathrm{ADC}(3)$ spectrum, which is slightly more peaked. According to EOM-CCSD, the most intense peak, just above $2 \mathrm{eV}$, corresponds to the transition to an excited state involving the two carbonyl LP's and a Rydberg state (state $\left.6 \mathrm{~A}^{\prime}\right)$. CAM-B3LYP provides same indications as the excited states in this region are deriving from the interaction between $4 \pi$ and several $n \rightarrow$ Rydberg states, the most intense ESA transitions involving a $\mathrm{n} \rightarrow$ Rydberg state (the same as in CCSD).

All methods examined here predict that the strongest transitions are found between 3 and $4 \mathrm{eV}$; however, the spectra differ in the number of intense transitions found in this region. For example, CAM-B3LYP yields two strong peaks, whereas, according to $\operatorname{ADC}(3)$, seven fairly intense transitions contribute to the peak. There is a general agreement concerning the relative intensity of this band with respect to those falling below $3 \mathrm{eV}$, that is, roughly six times larger. The band is due to the $\mathbf{1} \pi \rightarrow \mathbf{5} \boldsymbol{\pi}$ transition, mixed with transitions to states with HOMO $\rightarrow$ Rydberg character. The degree of mixing, which affects the number of "active" transitions, depends on the method considered. Nevertheless, a similar intensity of this band in all spectra indicates that the $\mathbf{1} \pi \rightarrow \mathbf{5} \pi$ 
transition is the one carrying the oscillator strength and that the role of double excitations is not significant.

This conclusion is confirmed by comparison with the ESA spectrum of the $1 \boldsymbol{\pi}$ from a recent RASPT 2 study, ${ }^{15}$ which (see computational details) provides the best RASPT2 theoretical reference of excitation energies and transition dipole moments for the singlet manifold of $\pi \pi^{*}$ states available until now. The RASPT2 spectrum has been reproduced in Figure 5 to ease the discussion. Notably, despite the absence of the Rydberg states contributions, motivating the smaller number of peaks, the position and relative intensity of the main bands are similar to that predicted by TD-CAM-B3LYP, EOM-CCSD, and EOMCC3. $A D C(2)$ and $\operatorname{ADC}(2)-x$, though also predicting the most prominent band above $3 \mathrm{eV}$, yield instead less structured spectra in the low-energy region.

It is important to keep in mind that any analysis of the ESA region $\geq 4 \mathrm{eV}$, both in the experiments and in calculations, can be problematic in the proximity to the first ionization threshold of uracil, which would make two photon ionization processes unavoidable.

3.2.2. ESA at 1n Stationary Points. As anticipated in Section 2, we also computed, at the EOM-CCSD level, the ESA at different 1 n stationary points obtained using different levels of theory. Moreover, as shown in Figure 6, we compare ESA spectra obtained at the planar (pseudo-minimum) and non-planar (minimum) EOM-CCSD geometries. EOMCCSD, CAM-B3LYP, and ADC(3) were used, which, as discussed earlier, provided ESA spectra similar to the EOM$\mathrm{CC} 3$ ones at the FC geometry. The spectra obtained at the two additional geometries R4 and R5, the latter exhibiting a noticeable out-of-plane distortion of the $\mathrm{C} 4 \mathrm{O} 8$ carbonyl group, are shown in Figure S5 in Supporting Information. All spectra exhibit a modest dependence on the chosen minima, though some differences can be found (see also Tables S14, S15, and S17 in Supporting Information).

Here, we shall focus our analysis on the results obtained at the EOM-CCSD planar and non-planar stationary points (cf. Figure 2), as shown in Figure 6.

Starting from the planar geometry, EOM-CCSD predicts a first absorption band in the region $2.2-3.2 \mathrm{eV}$, with an intense "central" peak just below $3 \mathrm{eV}$ (due to two close lying transitions) accompanied by less intense features on the redshifted and on the blue-shifted side. The lowest energy one, at $\sim 2.2 \mathrm{eV}$, is assigned to the $\mathbf{1 n} \rightarrow \mathbf{2 n}$ transition. The most intense one, at $2.91 \mathrm{eV}$, and the one at $3.16 \mathrm{eV}$ are assigned to the transitions from $1 n$ to two excited states that can be described as excitations from the combination of the LP on the two carbonyl groups to the lowest and second lowest energy $\pi^{*}$ orbitals. A fairly intense contribution to the $2.9 \mathrm{eV}$ band comes from the transition, just below $2.9 \mathrm{eV}$, to a $\pi$-Rydberg excited state. A series of closely lying transitions of moderate intensity contribute to the band at $\sim 4.2 \mathrm{eV}$, whereas two very strong peaks dominate the relatively intense band at $5 \mathrm{eV}$. They are due to transitions to states $19 \mathrm{~A}^{\prime \prime}$ and $20 \mathrm{~A}^{\prime \prime}$, both of Rydberg character.

The CAM-B3LYP spectra are different with respect to the CCSD ones, though the general pattern is somewhat similar, with three main peaks below $4 \mathrm{eV}$, the central one being the most intense in this region. However, their intensity is almost three times smaller than the one predicted by EOM-CCSD, confirming the trends highlighted in the FC region. The peaks are red-shifted with respect to EOM-CCSD by a few tenths of electron Volts, the central peak (the largest) falling at $\sim 2.5 \mathrm{eV}$.
They are assigned to the transition from $1 n$ to other $n \pi^{*}$ excited states, in line with the assignment derived from EOMCCSD. However, in this region, at variance from the EOMCCSD peak at $2.9 \mathrm{eV}$, no ESA to $\pi$-Rydberg excited state is found.

The spectra computed at the CAM-B3LYP 1n minimum are similar to those just described (see Figure S3).

$\mathrm{Up}$ to $4 \mathrm{eV}$, the ESA spectrum computed at the $\mathrm{ADC}(3)$ level is very similar to the one described above for CAMB3LYP but for a small, almost uniform, blue-shift. Above 4.5 $\mathrm{eV}$, the comparison between the three methods, in particular with CAM-B3LYP, is complicated by the possible occurrence of resonances. Indeed, in Hartree-Fock and TD-DFT quadratic response theory, ${ }^{59,60}$ computing the transition strength between two excited states involves the solution of a linear set of response equations of the form ${ }^{59,60}$

$$
\left[\mathbf{E}-\left(\omega_{f}-\omega_{i}\right) \mathbf{S}\right] \mathbf{N}^{A}\left(\omega_{f}-\omega_{i}\right)=\mathbf{A}
$$

at values of the energy corresponding to the ESA excitations, $\left(\omega_{f}-\omega_{i}\right)$. Since the OPA excitation energies are obtained solving the generalized eigenvalue equation

$$
(\mathbf{E}-\omega \mathbf{S}) \mathbf{X}=0
$$

it is clear that eq 3 may diverge if the energy difference between the states $i$ and $f,\left(\omega_{f}-\omega_{i}\right)$, is close to a one-photon excitation.

As highlighted by the gray spectrum in the mid panels of Figure 6, two transitions (depicted as dashed gray) are obtained at around $5 \mathrm{eV}$ with suspiciously large (relatively speaking) intensities. At the planar 1n geometry, one is at 5.03 $\mathrm{eV}$ with $f=0.15$ and the other at $5.15 \mathrm{eV}$ with $f=0.07$. At the non-planar geometry, they occur at $4.75 \mathrm{eV}$ with $f=0.17$ and $5.08 \mathrm{eV}$ with $f=0.16$. This could be an indication that we are in the proximity of a resonant frequency. At the $\mathbf{1 n}$ planar geometry, we obtain OPA excitations at $3.87,4.80$, and 5.48 $\mathrm{eV}$, whereas at the non-planar geometry, the same transitions are at $3.81,4.76$, and $5.47 \mathrm{eV}$.

The CAM-B3LYP profiles in blue have therefore been obtained excluding these values from the broadening. For $\mathrm{ADC}(3)$, no values above 4.5 (non-planar) -4.9 (planar) $\mathrm{eV}$ could be obtained due to convergence difficulties.

Finally, the main ESA peaks computed for all three methods at the non-planar minimum of $\mathbf{1 n}$ are similar to those just described for the planar one, only slightly more spread out. This is not surprising since the deviation from planarity is very small.

3.2.3. ESA at $1 \pi$ Stationary Point. Calculations using different methods ${ }^{28,36}$ show that a barrierless path on the PES of the $1 \pi$ state connects the FC point with a CI with GS, giving an account of the subpicosecond features revealed by timeresolved experiments for uracil both in the gas phase and in solution. ${ }^{12,27,28,31,61}$

On the other hand, analysis of the PES shows that a steep path connects the FC point with a low-energy gradient region, where the pyrimidine ring keeps a planar geometry. ${ }^{12,28,31}$ The motion to the CI involves loss of planarity, with pyramidalization of $\mathrm{C} 5$ and out-of-plane motion of H5, i.e. the $\mathrm{H}$ atom bonded to C5. The first part of this path is not very steep and a low-gradient non-planar pseudo-minimum is predicted by several methods. ${ }^{12,28,31,62}$

In order to obtain insights on the variation of the ESA associated to the motion of the wavepacket on the $1 \pi$ PES, we 

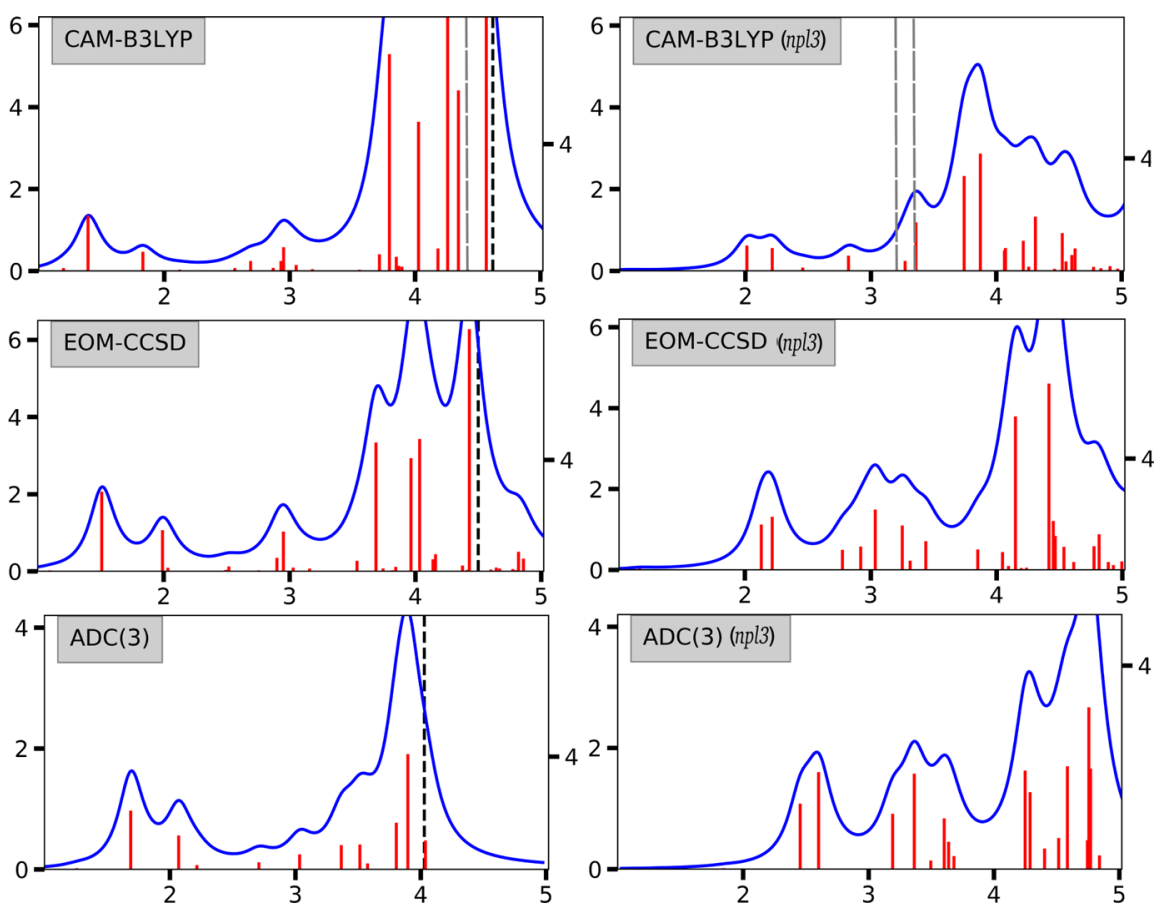

Figure 7. Uracil. ESA spectra from the lowest excited (bright) state at the optimized planar geometry (left panels) and at the npl3 geometry (right panel) of $\mathbf{1} \boldsymbol{\pi}$, for three different electronic structure methods. A vertical black dashed line, if present, corresponds to the estimated first IE of the excited state. In abscissa, we report the excitation energy in electron Volts and in left ordinate, the decadic molar extinction coefficient, ${ }^{42} \epsilon(\omega) \times$ $10^{-3}$, in units $\mathrm{M}^{-1} \mathrm{~cm}^{-1}$. On the right ordinate is the oscillator strength, $f \times 10^{2}$.

have thus computed the ESA at different representative planar and non-planar structures (see Figures 7 and S6).

We start our analysis from the pseudo-minimum (cf. Figure 2) optimized at the EOM-CCSD level forcing uracil to keep a planar geometry. The ESA spectra for the three electronic structure methods at this geometry are shown on the left panels of Figure 7.

The spectra predicted by EOM-CCSD and CAM-B3LYP are almost superimposable up to $3.5 \mathrm{eV}$, though the intensities predicted by EOM-CCSD are almost twice as large and, on an average, the peaks are slightly blue-shifted. There are two transitions at $\sim 1.5$ and $\sim 2 \mathrm{eV}$, which, according to both methods, are assigned to $1 \pi \rightarrow 2 \pi$ and $1 \pi \rightarrow 3 \pi$.

A cluster of transitions is then predicted at $\sim 3 \mathrm{eV}$, though significantly less intense than the peaks falling in this region for 1n, deriving from the mixing between $4 \pi$ and several $n \rightarrow$ Rydberg states.

Finally, both methods predict that several intense transitions lie at $4 \mathrm{eV}$. However, as also observed for the 1 n state, the analysis of the high-energy part of the CAM-B3LYP spectrum is complicated by the appearance of one transition, at $4.39 \mathrm{eV}$, (depicted as dashed gray) whose intensity is very large $(f=$ 0.62 ), and likely due to problems with resonant one-photon energies, as described in the previous section. Actually, the $\mathbf{1} \boldsymbol{\pi}$ excitation energy at the $\mathbf{1} \boldsymbol{\pi}$ pseudo-minimum is $4.49 \mathrm{eV}$. The blue spectrum in Figure 7 was therefore drawn excluding this transition, and it shows a number of relatively intense transitions ( 4 or 5), of which the two at $\sim 3.80$ and $\sim 4 \mathrm{eV}$ are assigned to the $\mathbf{1} \boldsymbol{\pi} \rightarrow \mathbf{5} \boldsymbol{\pi}$ transition mixed with HOMO $\rightarrow$ Rydberg character. The band peaks at around $\epsilon=15,000 \mathrm{M}^{-1}$ $\mathrm{cm}^{-1}$.

According to EOM-CCSD, at least four strong transitions lie in this region, including one at $\sim 4.5 \mathrm{eV}$. Two of them fall below $4 \mathrm{eV}$ and are assigned to the process leading from $1 \boldsymbol{\pi}$ to two HOMO/Rydberg excited states. The following one is due to the $1 \pi \rightarrow 5 \pi$ transition and, finally, the one on the blue to another $\pi \pi^{*}$ state involving the excitation from a low-lying $\pi$ orbital to the LUMO.

Also in this case, the ESA spectrum computed by $\mathrm{ADC}(3)$ is similar to the one of CAM-B3LYP up to $\sim 4 \mathrm{eV}$. Convergence problems were encountered at higher energies and no transitions could therefore be obtained $>4.0 \mathrm{eV}$.

For what concerns non-planar structures, independently of the methods, the spectra (see right panel of Figure 7 for npl3 and Figure S6 for npl1 and npl2) exhibit some common trends with respect to those obtained for the planar one. First, the lowest energy peaks are blue-shifted, falling, for npl3, above 2 $\mathrm{eV}$. Then, the number of transitions with significant oscillator strength increases. At non-planar geometries, $\mathrm{n} \pi^{*}$ and $\pi \pi^{*}$ mix, so that some transitions are no longer forbidden by symmetry and share oscillator strength. As a consequence, for npl3, the computed spectra suggest the existence of a broad absorption band peaking just above $2 \mathrm{eV}$ (i.e., $\sim 600 \mathrm{~nm}$ ), around $3 \mathrm{eV}$ (i.e., $\sim 400 \mathrm{~nm}$ ), and then between 4 and $5 \mathrm{eV}(270-250 \mathrm{~nm})$. Once again, CAM-B3LYP exhibits two transitions, at 3.09 and $3.24 \mathrm{eV}$, of very high intensity, in particular the first one. We again attribute them to resonances and exclude them from the spectral simulation, as shown in Figure 7. Indeed, the OPA excitation energy to $\mathbf{1} \boldsymbol{\pi}$ at $\mathrm{npl} 3$ structure is $3.08 \mathrm{eV}$, and the transition whose intensity is strongly overestimated falls at 3.09 eV.

\section{DISCUSSION}

Uracil has become a classical benchmark for electronic quantum mechanical methods. ${ }^{28,51}$ In addition to its biochemical relevance, its chemical structure and photophysical properties allow investigating several of common features of many families of compounds. We are indeed in the 


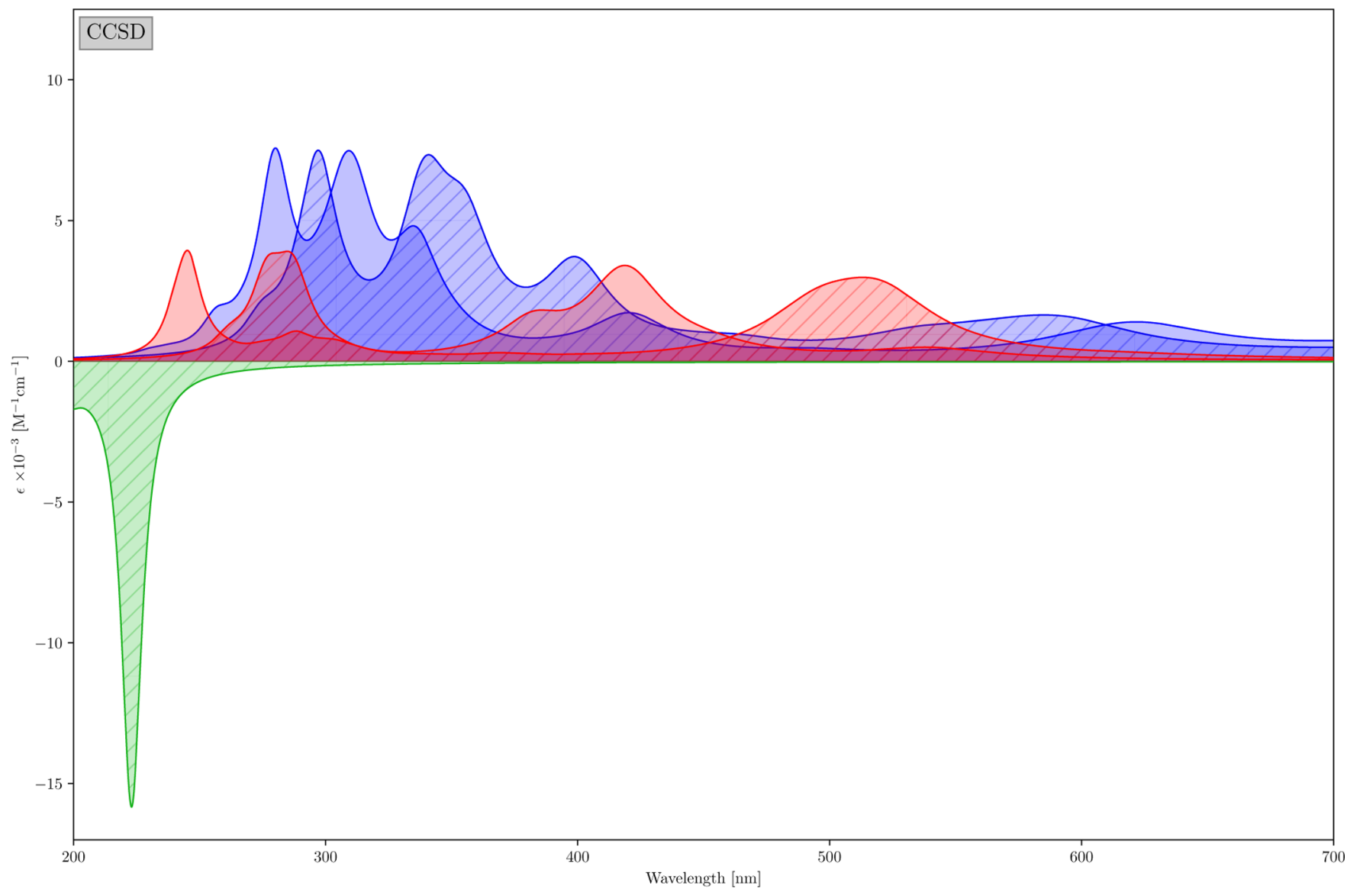

Figure 8. Uracil. Simulation of the pump-probe experiment at the CCSD level. Striped patterns correspond to the FC results (zero time delay). Green is the GS, red is the $1 \mathrm{n}$, and blue is the $1 \pi$ state.

presence of an heterocyclic compound, with two asymmetric exocyclic substituents (the carbonyl groups) heavily conjugated with the ring $\pi$ system. As a consequence, several different excited states with $\pi \pi^{*}$ and $\mathrm{n} \pi^{*}$ character are very close in the FC region, their interplay depending on the molecule structural rearrangements. Actually, the main nonradiative decay pathway involves a large deformation of the ring planarity, strongly coupling many excited states. ${ }^{27,28,58}$ Moreover, even the interaction with $\pi \sigma^{*}$ states cannot be discarded. For all these reasons, the study of the optical properties of uracil provides a stringent test for the performances of the computational methods tested in this paper, namely EOM-CCSD, EOM-CC3, TD-CAM-B3LYP, $\operatorname{ADC}(2), \operatorname{ADC}(2)-x$, and $\operatorname{ADC}(3)$.

Confirming previous indications, the different methods provide a fairly similar picture of the lowest energy bright transitions of the OPA spectrum. ${ }^{28}$ On the other hand, it is clear that, overall, the EOM-CCSD, CAM-B3LYP, and $\mathrm{ADC}(3)$ spectra are rather close, with three main "bands" peaking roughly at $\geq 5, \leq 7$, and $\leq 8 \mathrm{eV}$, the two first bands having similar intensity and the last one slightly more intense. As discussed in Section 3, the picture obtained from EOMCCSD, EOM-CC3, CAM-B3LYP, and ADC(3) appears to be fully consistent with the available experimental vapor phase spectra. ${ }^{53} \mathrm{ADC}(2)$ provides a rather different spectral pattern of the high-energy region, with the several moderately intense transitions between 6 and $7 \mathrm{eV}$, which is, nonetheless, also compatible with the experiments. Finally, the $\mathrm{ADC}(2)-\mathrm{x}$ spectra are significantly red-shifted with respect to the others and with respect to the experimental spectrum. The agreement between the six methods tested is instead much worse for what concerns the location of the dark $\mathrm{n} \pi^{*}$ transitions. In particular, the methods investigated provide rather different values for the energy gap between $1 \boldsymbol{\pi}$ and $1 \mathrm{n}$, though the estimates of EOMCCSD, EOM-CC3, and CAM-B3LYP are pretty similar (0.35-0.39 eV with 1 more stable). The energy difference at the FC point between $\mathbf{1} \boldsymbol{\pi}$ and $1 \mathrm{n}$ can remarkably affect the possible population transfer between these two states. As a consequence, the outcome of any dynamical simulation of this process would depend on the underlying electronic structure method.

For what concerns the main focus of our study, that is, the calculations of the ESA, EOM-CCSD, EOM-CC3, TD-CAMB3LYP, and ADC(3) yield similar ESA spectra in the FC region, for both $1 \pi$ and $1 \mathrm{n}$, though EOM-CCSD predicts, on an average, twice as large intensities. Actually, even a modest broadening would make extremely difficult to distinguish between TD-CAM-B3LYP and EOM-CC3 spectra up to $3 \mathrm{eV}$. There is also a fair agreement between the four methods in the 3-4 eV region, though, on an average, the TD-CAM-B3LYP spectra are less rich in that region (see below). Also in the case of $\mathrm{ESA}$, the $\mathrm{ADC}(2)$ and $\mathrm{ADC}(2)$-x predictions are different from those of the other four methods: the ESA of $1 n$ and $\mathbf{1} \pi$ are red-shifted and their pattern of intensity is different. On the other hand, some common trends can be recognized. For 1n, under $4 \mathrm{eV}$, a single ESA band, due to two close-lying transition, is predicted, whose maximum shifts from $<2$ to 2.5 $\mathrm{eV}$ depending on the method. For $\mathbf{1} \boldsymbol{\pi}$, three main bands are 
predicted, peaking roughly at $1.5,2.5$, and $3.5 \mathrm{eV}$, the last one being the most intense.

Our calculations of ESA in the stationary and pseudostationary points of $1 \mathrm{n}$ and $\mathbf{1} \boldsymbol{\pi}$ confirm the general agreement between EOM-CCSD and TD-CAM-B3LYP spectra, though the former ones are significantly more intense. On the other hand, the discrepancy between these two methods increases in the high-energy region.

This study provides encouraging indications on the reliability of TD-CAM-B3LYP, especially up to $4 \mathrm{eV}$. However, interestingly, TD-CAM-B3LYP spectra fairly agree with the EOM-CCSD and EOM-CC3 ones, also in the high-energy region, close to the IP of uracil, a region in which some failures of TD-DFT could be expected. On an average, we register that the TD-CAM-B3LYP spectra are "less rich" above $4 \mathrm{eV}$, that is, the number of intense transitions decreases with respect to the other methods. This could be due to some difficulties in treating the coupling between higher-lying Rydberg states. Actually, at least for the ESA from 1 n and $1 \boldsymbol{\pi}$ minima, the comparison between TD-CAM-B3LYP and the other methods is, in this region, made more difficult by the risk of obtaining nonphysically high values of the transition strength due to (possible) divergences, in the proximity of a resonant frequency, in the linear response term that enters the expression of the TD-DFT transition moment between two excited states. Possible strategies to bypass this problem, falling outside the scope of the present study, could be to use MOM instead of standard DFT to compute the ESA spectra or to adopt suitable damping procedures in the proximity of the resonance.

Double excitations should play a minor role, at least in the energy range here investigated. This is also shown by the reasonable agreement between EOM-CCSD and EOM-CC3 spectra and, at least for what concerns $1 \pi$ in the FC region, the strong similarity between single-reference based methods and RASPT2.

On the other hand, test calculations, reported in Supporting Information (see Figure S7), using B3LYP indicate that, as it could be expected, the quality of the results depends on the choice of the functional. B3LYP and CAM-B3LYP spectra exhibit overall a similar shape for $\mathbf{1} \boldsymbol{\pi}$, but the former are on an average red-shifted. As a consequence, for $1 \pi$ the B3LYP spectra are consistent with those by the other methods. For 1n, on the other hand, two (relatively) strong absorption peaks are predicted well below $2 \mathrm{eV}$, in noticeable disagreement with EOM-CC3. Moreover, the intensity of the band at $4 \mathrm{eV}$ is much larger than for CAM-B3LYP.

To the best of our knowledge, ESA experimental spectra in the gas phase are not available, whereas some indications have been obtained in water. Solvent effect is missing in our calculations, and it will be the subject of a forthcoming study. On the other hand, previous work shows that solvents able to establish hydrogen bonds weakly affect $\pi \pi^{*}$ transitions and strongly destabilize $\mathrm{n} \pi^{*} .^{28,62}$ In the hypothesis that these effects are, roughly, the same for these kind of transitions for any $\pi \pi^{*}$ and for any $\mathrm{n} \pi^{*}$, ESA should not be dramatically affected since it is largely dominated by $\pi \pi^{*} \rightarrow \pi \pi^{*}$ and $\mathrm{n} \pi^{*}$ $\rightarrow \mathrm{n} \pi^{*}$ absorption.

Under these assumptions, our results appear consistent with the available experiments. To facilitate the comparison with the experimental results, which are usually discussed on the wavelength scale, we report our results on this scale in Figure 8. The $\mathrm{S}_{0}$ OPA is expressed as a negative signal, to simulate GS bleaching. Please note that the experimental pump-probe spectrum, and the experimental TA spectra also measure stimulated emission, which is not considered in our treatment. In the fluorescence spectrum of uracil, a broad spectrum peaking at $310 \mathrm{~nm}$, associated to emission from $1 \boldsymbol{\pi}$, is present and would provide a negative contribution to the ESA in that region. ${ }^{63}$

For $1 \pi$ in the FC region we predict, according to CCSD, four broad ESA bands, peaking roughly just below $600 \mathrm{~nm}$, at $\sim 400, \sim 355$, and $300 \mathrm{~nm}$. When going toward the minimum, the lowest energy band red shifts slightly, whereas the remaining part of the spectrum blue shifts, exhibiting a broad feature peaking at $\sim 410 \mathrm{~nm}$ and maxima at $\sim 355, \sim 300$, and $\sim 275 \mathrm{~nm}$.

A broad ESA around $600 \mathrm{~nm}$ is usually associated to nucleobases in solution, ${ }^{64}$ in agreement with our predictions (considering the effect of stimulated emission in the blue wing of the spectrum). Though our calculations predict significant ESA also below $300 \mathrm{~nm}$, the intensity is smaller than that computed in the OPA spectrum. This indication is in line with the observation of negative induced absorbance (bleaching) in the TA spectra measured at $250 \mathrm{~nm}$ until the GS is repopulated. $^{65}$

On the other hand, the contribution of the ESA would modify the GS bleaching signal, even in the absence of any significant repopulation of the ground electronic state. The subtle dependence of the spectral signal on the dynamics of several intertangled excited states makes indeed its interpretation very complicated, and calculations can significantly help it.

For what concerns $1 \mathrm{n}$, we predict a peak around $500 \mathrm{~nm}$ in the FC region, and rather intense bands, with maxima at $~ 390$ and $\sim 420 \mathrm{~nm}$, in its minimum. Interestingly, an intense band at $\sim 300 \mathrm{~nm}$ is predicted, blue-shifting at $\sim 250 \mathrm{~nm}$ in the minimum. The first band would thus interfere with the stimulated emission peak, while the latter with the GS bleaching.

This result would be consistent with the features of the longliving dark states evidenced for 1-cyclohexyl-uracil in different solvents ${ }^{29}$ which absorbs in the $300-450 \mathrm{~nm}$ range (and not for longer wavelengths). This state has been indeed assigned to $\mathbf{1 n},{ }^{29}$ though its involvement in pyrimidine photophysics is highly debated. ${ }^{28}$

As discussed in Section 3, CAM-B3LYP would provide similar pump-probe spectra to those of CCSD, but the lowest energy positive peaks would be slightly red-shifted.

From a more general point of view, even though, on an average, ESA from $1 \boldsymbol{\pi}$ is larger, our calculations clearly indicate that ESA from $n \pi^{*}$ can also be significant. Actually, there are some energy windows for which ESA from $1 \mathbf{n}$ is significantly larger than that from $1 \boldsymbol{\pi}$.

In this respect, our calculations on the non-planar structures show that, as it could be expected, the mixing between states of different symmetry leads to much richer and broader spectra, and a proper treatment of the states that are dark in the FC region is critical for a correct reproduction of the spectra.

In this contribution, we did not consider vibronic effects, an aspect actually rarely addressed for ESA. ${ }^{66}$ As far as uracil is concerned, even in the gas phase, the OPA is broad and structureless as a result of not only the remarkable displacement of the equilibrium geometries and strong Duschinsky effects $^{67}$ but also of $\mathbf{1} \boldsymbol{\pi} / \mathbf{1 n}$ inter-state couplings (investigated for thymine in ref 68 ). It is reasonable to assume that these 
effects do play a similar or even larger role in the ESA spectra. More in general, it should be emphasized that we did not consider dynamical effects. In fact, we considered "idealized" situations in which the photoexcited wavepacket is on just one electronic state $(\mathbf{1} \boldsymbol{\pi}$ or $\mathbf{1 n})$ per time. In reality, at least in the ultrafast time regime, the wavepacket is expected to move simultaneously on the two states. ${ }^{68,69}$

\section{CONCLUSIONS}

In this study, we report a critical comparison between the absorption spectra, OPA and ESA, computed for uracil in the gas phase using different computational approaches, focusing in particular on the ESA of the two lowest energy excited states, one $\mathrm{n} \pi^{*}$ and one $\pi \pi^{*}$, in different critical regions of the PES.

We provide a thorough computational analysis of the OPA spectrum of uracil in the gas phase, not only assigning the most intense transitions up to $9 \mathrm{eV}$ but also locating the $\mathrm{n} \pi^{*}$ transitions and the transitions involving Rydberg states. We also obtain very interesting indications on the performances of different methods in describing the ESA of a key heteroatomic molecule.

From the methodological point of view, CAM-B3LYP and EOM-CCSD are in fairly good agreement with the computationally more expensive EOM-CC3 [and $\mathrm{ADC}(3)$ ] or with available RASPT2 spectra, for what concerns both dark and bright transitions, even in the high-energy region, where the weight of double excitations could be larger.

At the same time, we obtain some indications on the possible problems of the present implementation of TD-DFT in some spectral regions.

Spectra computed with $\operatorname{ADC}(2)$ and $\operatorname{ADC}(2)-x$ are rather similar, with $\mathrm{ADC}(2)-\mathrm{x}$ showing lower intensities than $\operatorname{ADC}(2)$. The $A D C(2)-x$ method provides OPA and ESA spectra that are red-shifted compared to all other methods. This general lowering of the excitation energies was clearly highlighted in the review of Dreuw and Wormit ${ }^{70}$ and attributed to an unbalanced description of the excitation spectrum. The lowering has been observed in several benchmarking studies. ${ }^{26,71,72}$ It remains unclear whether $\mathrm{ADC}(2)$-x actually provides a significant improvement over $\operatorname{ADC}(2)$, despite its higher computational cost.

We also show that the ESA from a state that is dark in OPA, as $1 \mathbf{n}$, cannot be neglected and that in some energy scale, it would rule the ESA spectrum. Moreover, we have the first indications on the expected changes in the spectra when the wavepacket moves away from the FC region toward the minima and the crossing region. In the latter case, the departure from planarity and the coupling between transitions with different symmetry leads to a significant broadening of the spectral shape.

Response methods appear to be appealing choice since they can, in principle, treat excited states of different symmetry on the same footing. This possibility is very important to interpret the contribution to the spectral signals coming from the nonplanar region, which dominates the ultrafast GS recovery in many molecules, as uracil, and for which reduction/selection of the right active space is more cumbersome. In this respect, the novel, very efficient, implementation of the EOM-CC3 method used here ${ }^{25}$ is a particularly attractive addition to the set of coupled cluster methods currently available.

The good performances of TD-CAM-B3LYP are also comforting, confirming that this approach is very cost-effective and putting on a firmer ground its eventual use to study larger molecular systems.

\section{ASSOCIATED CONTENT}

\section{Supporting Information}

The Supporting Information is available free of charge at https://pubs.acs.org/doi/10.1021/acs.jctc.0c01150.

Raw spectral data, natural transition orbitals, additional spectra, and Cartesian coordinates of the different structures (PDF)

\section{AUTHOR INFORMATION}

\section{Corresponding Authors}

Sonia Coriani - DTU Chemistry, Technical University of Denmark, DK-2800 Kongens Lyngby, Denmark; Department of Chemistry, NTNU-Norwegian University of Science and Technology, N-7491 Trondheim, Norway; (1) orcid.org/ 0000-0002-4487-897X; Email: soco@kemi.dtu.dk

Roberto Improta - Istituto di Biostrutture e BioimmaginiCNR, I-80134 Napoli, Italy; ㅇo orcid.org/0000-0003-1004195X; Email: robimp@unina.it

\section{Authors}

Daniil A. Fedotov - DTU Chemistry, Technical University of Denmark, DK-2800 Kongens Lyngby, Denmark; (1) orcid.org/0000-0001-5019-2096

Alexander C. Paul - Department of Chemistry, NTNUNorwegian University of Science and Technology, N-7491 Trondheim, Norway; orcid.org/0000-0002-7547-445X

Paolo Posocco - Dipartimento di Scienze Chimiche e Farmaceutiche, Università degli Studi di Trieste, I-34127 Trieste, Italy

Fabrizio Santoro - Istituto di Chimica dei Composti Organometallici (ICCOM-CNR), Area della Ricerca del CNR, I-56124 Pisa, Italy; 이이.org/0000-0003-44022685

Marco Garavelli - Department of Industrial Chemistry "Toso Montanari”, Università degli Studi di Bologna, I-40126 Bologna, Italy; (1) orcid.org/0000-0002-0796-289X

Henrik Koch - Scuola Normale Superiore, I-56100 Pisa, Italy; Department of Chemistry, NTNU-Norwegian University of Science and Technology, N-7491 Trondheim, Norway; o orcid.org/0000-0002-8367-8727

Complete contact information is available at:

https://pubs.acs.org/10.1021/acs.jctc.0c01150

\section{Notes}

The authors declare no competing financial interest.

\section{ACKNOWLEDGMENTS}

This work has received funding from the European Union's Horizon 2020 research and innovation program under the Marie Skłodowska-Curie European Training Network [grant agreement no. 765739 (COSINE-COmputational Spectroscopy In Natural sciences and Engineering, D.A.F., A.C.P., H.K., and S.C.) and no. 765266 (LightDyNAmics, R.I., F.S., and M.G.)]. S.C. acknowledges financial support from the Independent Research Fund Denmark-DFF-FNU RP2 (grant no. 7014-00258B). A.C.P., S.C., and H.K. acknowledge the Research Council of Norway through FRINATEK projects 263110 and 275506. 


\section{REFERENCES}

(1) Maiuri, M.; Garavelli, M.; Cerullo, G. Ultrafast Spectroscopy: State of the Art and Open Challenges. J. Am. Chem. Soc. 2020, 142, $3-15$.

(2) Oliver, T. A. A. Recent advances in multidimensional ultrafast spectroscopy. R. Soc. Open Sci. 2018, 5, 171425.

(3) Raavi, S. S. K.; Biswas, C. Digital Encyclopedia of Applied Physics; American Cancer Society, 2019; pp 1-49.

(4) Berera, R.; van Grondelle, R.; Kennis, J. T. M. Ultrafast transient absorption spectroscopy: principles and application to photosynthetic systems. Photosynth. Res. 2009, 101, 105-118.

(5) Ruckebusch, C.; Sliwa, M.; Pernot, P.; de Juan, A.; Tauler, R. Comprehensive data analysis of femtosecond transient absorption spectra: A review. J. Photochem. Photobiol., C 2012, 13, 1-27.

(6) Fischer, S. A.; Cramer, C. J.; Govind, N. Excited State Absorption from Real-Time Time-Dependent Density Functional Theory. J. Chem. Theory Comput. 2015, 11, 4294-4303.

(7) Cronstrand, P.; Christiansen, O.; Norman, P.; Ågren, H. Theoretical calculations of excited state absorption. Phys. Chem. Chem. Phys. 2000, 2, 5357-5363.

(8) Ling, S.; Schumacher, S.; Galbraith, I.; Paterson, M. J. Excitedstate absorption of conjugated polymers in the near-infrared and visible: a computational study of oligofluorenes. J. Phys. Chem. C 2013, 117, 6889-6895.

(9) Denis, J.-C.; Ruseckas, A.; Hedley, G. J.; Matheson, A. B.; Paterson, M. J.; Turnbull, G. A.; Samuel, I. D. W.; Galbraith, I. Selftrapping and excited state absorption in fluorene homo-polymer and copolymers with benzothiadiazole and tri-phenylamine. Phys. Chem. Chem. Phys. 2016, 18, 21937-21948.

(10) Bowman, D. N.; Asher, J. C.; Fischer, S. A.; Cramer, C. J.; Govind, N. Excited-state absorption in tetrapyridyl porphyrins: comparing real-time and quadratic-response time-dependent density functional theory. Phys. Chem. Chem. Phys. 2017, 19, 27452-27462.

(11) Giussani, A.; Segarra-Martí, J.; Nenov, A.; Rivalta, I.; Tolomelli, A.; Mukamel, S.; Garavelli, M. Spectroscopic fingerprints of DNA/ RNA pyrimidine nucleobases in third-order nonlinear electronic spectra. Theor. Chem. Acc. 2016, 135, 121.

(12) Pepino, A. J.; Segarra-Martí, J.; Nenov, A.; Improta, R.; Garavelli, M. Resolving Ultrafast Photoinduced Deactivations in Water-Solvated Pyrimidine Nucleosides. J. Phys. Chem. Lett. 2017, 8, 1777-1783.

(13) Martínez-Fernández, L.; Pepino, A. J.; Segarra-Martí, J.; Jovaišaite, J.; Vaya, I.; Nenov, A.; Markovitsi, D.; Gustavsson, T.; Banyasz, A.; Garavelli, M.; Improta, R. Photophysics of Deoxycytidine and 5-Methyldeoxycytidine in Solution: A Comprehensive Picture by Quantum Mechanical Calculations and Femtosecond Fluorescence Spectroscopy. J. Am. Chem. Soc. 2017, 139, 7780-7791.

(14) Schmid, M.; Martinez-Fernandez, L.; Markovitsi, D.; Santoro, F.; Hache, F.; Improta, R.; Changenet, P. Unveiling Excited-State Chirality of Binaphthols by Femtosecond Circular Dichroism and Quantum Chemical Calculations. J. Phys. Chem. Lett. 2019, 10, 40894094.

(15) Jaiswal, V. K.; Segarra-Martí, J.; Marazzi, M.; Zvereva, E.; Assfeld, X.; Monari, A.; Garavelli, M.; Rivalta, I. First-principles characterization of the singlet excited state manifold in DNA/RNA nucleobases. Phys. Chem. Chem. Phys. 2020, 22, 15496-15508.

(16) González, L.; Escudero, D.; Serrano-Andrés, L. Progress and Challenges in the Calculation of Electronic Excited States. ChemPhysChem 2012, 13, 28-51.

(17) Gilbert, A. T. B.; Besley, N. A.; Gill, P. M. W. Self-Consistent Field Calculations of Excited States Using the Maximum Overlap Method (MOM) †. J. Phys. Chem. A 2008, 112, 13164-13171.

(18) Hait, D.; Head-Gordon, M. Excited State Orbital Optimization via Minimizing the Square of the Gradient: General Approach and Application to Singly and Doubly Excited States via Density Functional Theory. J. Chem. Theory Comput. 2020, 16, 1699-1710.

(19) Tsuru, S.; Vidal, M. L.; Pápai, M.; Krylov, A. I.; Møller, K. B.; Coriani, $\mathrm{S}$. Time-resolved near-edge X-ray absorption fine structure of pyrazine from electronic structure and nuclear wave packet dynamics simulations. J. Chem. Phys. 2019, 151, 124114.

(20) Vidal, M. L.; Krylov, A. I.; Coriani, S. Dyson orbitals within the fc-CVS-EOM-CCSD framework: theory and application to X-ray photoelectron spectroscopy of ground and excited states. Phys. Chem. Chem. Phys. 2020, 22, 2693-2703.

(21) Vidal, M. L.; Krylov, A. I.; Coriani, S. Correction: Dyson orbitals within the fc-CVS-EOM-CCSD framework: theory and application to X-ray photoelectron spectroscopy of ground and excited states. Phys. Chem. Chem. Phys. 2020, 22, 3744-3747.

(22) Stanton, J. F.; Bartlett, R. J. The equation of motion coupledcluster method. A systematic biorthogonal approach to molecular excitation energies, transition probabilities, and excited state properties. J. Chem. Phys. 1993, 98, 7029-7039.

(23) Bartlett, R. J. Coupled-cluster theory and its equation-ofmotion extensions. Wiley Interdiscip. Rev.: Comput. Mol. Sci. 2012, 2, $126-138$.

(24) Krylov, A. I. Equation-of-Motion Coupled-Cluster Methods for Open-Shell and Electronically Excited Species: The Hitchhiker's Guide to Fock Space. Annu. Rev. Phys. Chem. 2008, 59, 433-462.

(25) Paul, A. C.; Myhre, R. H.; Koch, H. New and Efficient Implementation of CC3. J. Chem. Theory Comput. 2021, 17, 117.

(26) Harbach, P. H. P.; Wormit, M.; Dreuw, A. The third-order algebraic diagrammatic construction method $(\operatorname{ADC}(3))$ for the polarization propagator for closed-shell molecules: Efficient implementation and benchmarking. J. Chem. Phys. 2014, 141, 064113.

(27) Barbatti, M.; Borin, A. C.; Ullrich, S. Photoinduced Phenomena in Nucleic Acids I: Nucleobases in the Gas Phase and in Solvents; Barbatti, M., Borin, C. A., Ullrich, S., Eds.; Springer International Publishing: Cham, Switzerland, 2015; Vol. 355; pp 1-32.

(28) Improta, R.; Santoro, F.; Blancafort, L. Quantum mechanical studies on the photophysics and the photochemistry of nucleic acids and nucleobases. Chem. Rev. 2016, 116, 3540-3593.

(29) Hare, P. M.; Crespo-Hernández, C. E.; Kohler, B. Solventdependent photophysics of 1-cyclohexyluracil: ultrafast branching in the initial bright state leads nonradiatively to the electronic ground state and a long-lived $\ln \pi^{*}$ state. J. Phys. Chem. B 2006, 110, 1864118650.

(30) Pilles, B. M.; Maerz, B.; Chen, J.; Bucher, D. B.; Gilch, P.; Kohler, B.; Zinth, W.; Fingerhut, B. P.; Schreier, W. J. Decay Pathways of Thymine Revisited. J. Phys. Chem. A 2018, 122, 48194828.

(31) Pepino, A. J.; Segarra-Martí, J.; Nenov, A.; Rivalta, I.; Improta, R.; Garavelli, M. UV-induced long-lived decays in solvated pyrimidine nucleosides resolved at the MS-CASPT2/MM level. Phys. Chem. Chem. Phys. 2018, 20, 6877-6890.

(32) Prokhorenko, V. I.; Picchiotti, A.; Pola, M.; Dijkstra, A. G.; Miller, R. J. D. New Insights into the Photophysics of DNA Nucleobases. J. Phys. Chem. Lett. 2016, 7, 4445.

(33) Peterson, K. A.; Dunning, T. H., Jr. Accurate correlation consistent basis sets for molecular core-valence correlation effects: The second row atoms $\mathrm{Al}-\mathrm{Ar}$, and the first row atoms $\mathrm{B}-\mathrm{Ne}$ revisited. J. Chem. Phys. 2002, 117, 10548-10560.

(34) Kaufmann, K.; Baumeister, W.; Jungen, M. Universal Gaussian basis sets for an optimum representation of Rydberg and continuum wavefunctions. J. Phys. B: At., Mol. Opt. Phys. 1989, 22, 2223-2240.

(35) Aquilante, F.; Autschbach, J.; Carlson, R. K.; Chibotaru, L. F.; Delcey, M. G.; De Vico, L.; Galván, I. F.; Ferré, N.; Frutos, L. M.; Gagliardi, L.; Garavelli, M.; Giussani, A.; Hoyer, C. E.; Li Manni, G.; Lischka, H.; Ma, D.; Malmqvist, P. Å.; Müller, T.; Nenov, A.; Olivucci, M.; Pedersen, T. B.; Peng, D.; Plasser, F.; Pritchard, B.; Reiher, M.; Rivalta, I.; Schapiro, I.; Segarra-Martí, J.; Stenrup, M.; Truhlar, D. G.; Ungur, L.; Valentini, A.; Vancoillie, S.; Veryazov, V.; Vysotskiy, V. P.; Weingart, O.; Zapata, F.; Lindh, R. Molcas 8: New capabilities for multiconfigurational quantum chemical calculations across the periodic table. J. Comput. Chem. 2016, 37, 506-541.

(36) Matsika, S. Radiationless Decay of Excited States of Uracil through Conical Intersections. J. Phys. Chem. A 2004, 108, 75847590 . 
(37) Frisch, M. J.; Trucks, G. W.; Schlegel, H. B.; Scuseria, G. E.; Robb, M. A.; Cheeseman, J. R.; Scalmani, G.; Barone, V.; Petersson, G. A.; Nakatsuji, H.; Li, X.; Caricato, M.; Marenich, A. V.; Bloino, J.; Janesko, B. G.; Gomperts, R.; Mennucci, B.; Hratchian, H. P.; Ortiz, J. V.; Izmaylov, A. F.; Sonnenberg, J. L.; Williams-Young, D.; Ding, F.; Lipparini, F.; Egidi, F.; Goings, J.; Peng, B.; Petrone, A.; Henderson, T.; Ranasinghe, D.; Zakrzewski, V. G.; Gao, J.; Rega, N.; Zheng, G.; Liang, W.; Hada, M.; Ehara, M.; Toyota, K.; Fukuda, R.; Hasegawa, J.; Ishida, M.; Nakajima, T.; Honda, Y.; Kitao, O.; Nakai, H.; Vreven, T.; Throssell, K.; Montgomery, J. A., Jr.; Peralta, J. E.; Ogliaro, F.; Bearpark, M. J.; Heyd, J. J.; Brothers, E. N.; Kudin, K. N.; Staroverov, V. N.; Keith, T. A.; Kobayashi, R.; Normand, J.; Raghavachari, K.; Rendell, A. P.; Burant, J. C.; Iyengar, S. S.; Tomasi, J.; Cossi, M.; Millam, J. M.; Klene, M.; Adamo, C.; Cammi, R.; Ochterski, J. W.; Martin, R. L.; Morokuma, K.; Farkas, O.; Foresman, J. B.; Fox, D. J. Gaussian 16, Revision C.01; Gaussian, Inc., 2016.

(38) Shao, Y.; Gan, Z.; Epifanovsky, E.; Gilbert, A. T. B.; Wormit, M.; Kussmann, J.; Lange, A. W.; Behn, A.; Deng, J.; Feng, X.; Ghosh, D.; Goldey, M.; Horn, P. R.; Jacobson, L. D.; Kaliman, I.; Khaliullin, R. Z.; Kuś, T.; Landau, A.; Liu, J.; Proynov, E. I.; Rhee, Y. M.; Richard, R. M.; Rohrdanz, M. A.; Steele, R. P.; Sundstrom, E. J.; Woodcock, H. L., III; Zimmerman, P. M.; Zuev, D.; Albrecht, B.; Alguire, E.; Austin, B.; Beran, G. J. O.; Bernard, Y. A.; Berquist, E.; Brandhorst, K.; Bravaya, K. B.; Brown, S. T.; Casanova, D.; Chang, C.M.; Chen, Y.; Chien, S. H.; Closser, K. D.; Crittenden, D. L.; Diedenhofen, M.; DiStasio, R. A., Jr.; Do, H.; Dutoi, A. D.; Edgar, R. G.; Fatehi, S.; Fusti-Molnar, L.; Ghysels, A.; Golubeva-Zadorozhnaya, A.; Gomes, J.; Hanson-Heine, M. W. D.; Harbach, P. H. P.; Hauser, A. W.; Hohenstein, E. G.; Holden, Z. C.; Jagau, T.-C.; Ji, H.; Kaduk, B.; Khistyaev, K.; Kim, J.; Kim, J.; King, R. A.; Klunzinger, P.; Kosenkov, D.; Kowalczyk, T.; Krauter, C. M.; Lao, K. U.; Laurent, A. D.; Lawler, K. V.; Levchenko, S. V.; Lin, C. Y.; Liu, F.; Livshits, E.; Lochan, R. C.; Luenser, A.; Manohar, P.; Manzer, S. F.; Mao, S.-P.; Mardirossian, N.; Marenich, A. V.; Maurer, S. A.; Mayhall, N. J.; Neuscamman, E.; Oana, C. M.; Olivares-Amaya, R.; O’Neill, D. P.; Parkhill, J. A.; Perrine, T. M.; Peverati, R.; Prociuk, A.; Rehn, D. R.; Rosta, E.; Russ, N. J.; Sharada, S. M.; Sharma, S.; Small, D. W.; Sodt, A.; Stein, T.; Stück, D.; Su, Y.-C.; Thom, A. J. W.; Tsuchimochi, T.; Vanovschi, V.; Vogt, L.; Vydrov, O.; Wang, T.; Watson, M. A.; Wenzel, J.; White, A.; Williams, C. F.; Yang, J.; Yeganeh, S.; Yost, S. R.; You, Z.-Q.; Zhang, I. Y.; Zhang, X.; Zhao, Y.; Brooks, B. R.; Chan, G. K. L.; Chipman, D. M.; Cramer, C. J.; Goddard, W. A., III; Gordon, M. S.; Hehre, W. J.; Klamt, A.; Schaefer, H. F., III; Schmidt, M. W.; Sherrill, C. D.; Truhlar, D. G.; Warshel, A.; Xu, X.; AspuruGuzik, A.; Baer, R.; Bell, A. T.; Besley, N. A.; Chai, J.-D.; Dreuw, A.; Dunietz, B. D.; Furlani, T. R.; Gwaltney, S. R.; Hsu, C.-P.; Jung, Y.; Kong, J.; Lambrecht, D. S.; Liang, W.; Ochsenfeld, C.; Rassolov, V. A.; Slipchenko, L. V.; Subotnik, J. E.; Van Voorhis, T.; Herbert, J. M.; Krylov, A. I.; Gill, P. M. W.; Head-Gordon, M. Advances in molecular quantum chemistry contained in the Q-Chem 4 program package. Mol. Phys. 2015, 113, 184-215.

(39) Stanton, J. F.; Gauss, J.; Cheng, L.; Harding, M. E.; Matthews, D. A.; Szalay, P. G. CFOUR, Coupled-Cluster Techniques for Computational Chemistry, a Quantum-Chemical Program Package. With contributions from Auer, A. A.; Bartlett, R. J.; Benedikt, U.; Berger, C.; Bernholdt, D. E.; Bomble, Y. J.; Christiansen, O.; Enge, F.; Faber, R.; Heckert, M.; Heun, O.; Hilgenberg, M.; Huber, C.; Jagau, T.-C.; Jonsson, D.; Jusélius, J.; Kirsch, T.; Klein, K.; Lauderdale, W. J.; Lipparini, F.; Metzroth, T.; Mück, L. A.; O’Neill, D. P.; Price, D. R.; Prochnow, E.; Puzzarini, C.; Ruud, K.; Schiffmann, F.; Schwalbach, W.; Simmons, C.; Stopkowicz, S.; Tajti, A.; Vázquez, J.; Wang, F.; Watts, J. D. and the integral packages MOLECULE (Almlöf, J.; Taylor, P.R.), PROPS (Taylor. P.R.), ABACUS (Helgaker, T.; Jensen, H. J. A.; Jørgensen, P.; Olsen, J.), and ECP routines by Mitin, A. V.; van Wüllen, C. For the current version, see http://www.cfour.de.

(40) Aidas, K.; Angeli, C.; Bak, K. L.; Bakken, V.; Bast, R.; Boman, L.; Christiansen, O.; Cimiraglia, R.; Coriani, S.; Dahle, P.; Dalskov, E. K.; Ekström, U.; Enevoldsen, T.; Eriksen, J. J.; Ettenhuber, P.; Fernández, B.; Ferrighi, L.; Fliegl, H.; Frediani, L.; Hald, K.; Halkier,
A.; Hättig, C.; Heiberg, H.; Helgaker, T.; Hennum, A. C.; Hettema, H.; Hjertenaes, E.; Høst, S.; Høyvik, I.-M.; Iozzi, M. F.; Jansík, B.; Jensen, H. J. A.; Jonsson, D.; Jørgensen, P.; Kauczor, J.; Kirpekar, S.; Kjaergaard, T.; Klopper, W.; Knecht, S.; Kobayashi, R.; Koch, H.; Kongsted, J.; Krapp, A.; Kristensen, K.; Ligabue, A.; Lutnaes, O. B.; Melo, J. I.; Mikkelsen, K. V.; Myhre, R. H.; Neiss, C.; Nielsen, C. B.; Norman, P.; Olsen, J.; Olsen, J. M. H.; Osted, A.; Packer, M. J.; Pawlowski, F.; Pedersen, T. B.; Provasi, P. F.; Reine, S.; Rinkevicius, Z.; Ruden, T. A.; Ruud, K.; Rybkin, V. V.; Sałek, P.; Samson, C. C. M.; de Merás, A. S.; Sauer, T.; Schimmelpfennig, B.; Sneskov, K.; Steindal, A. H.; Sylvester-Hvid, K. O.; Taylor, P. R.; Teale, A. M.; Tellgren, E. I.; Tew, D. P.; Thorvaldsen, A. J.; Thøgersen, L.; Vahtras, O.; Watson, M. A.; Wilson, D. J. D.; Ziolkowski, M.; Ågren, H. The Dalton Quantum Chemistry Program System. Wiley Interdiscip. Rev.: Comput. Mol. Sci. 2014, 4, 269.

(41) Folkestad, S. D.; Kjønstad, E. F.; Myhre, R. H.; Andersen, J. H.; Balbi, A.; Coriani, S.; Giovannini, T.; Goletto, L.; Haugland, T. S.; Hutcheson, A.; Høyvik, I.-M.; Moitra, T.; Paul, A. C.; Scavino, M.; Skeidsvoll, A. S.; Tveten, Å. H.; Koch, H. eT 1.0: An open source electronic structure program with emphasis on coupled cluster and multilevel methods. J. Chem. Phys. 2020, 152, 184103.

(42) Rizzo, A.; Coriani, S.; Ruud, K. Response function theory computational approaches to linear and non-linear optical spectroscopy. Computational Strategies for Spectroscopy: from Small Molecules to Nano Systems; John Wiley \& Sons Ltd., 2012; p 77.

(43) Stanton, J. F.; Gauss, J. A simple scheme for the direct calculation of ionization potentials with coupled-cluster theory that exploits established excitation energy methods. J. Chem. Phys. 1999, $111,8785-8788$

(44) Coriani, S.; Koch, H. Communication: X-ray absorption spectra and core-ionization potentials within a core-valence separated coupled cluster framework. J. Chem. Phys. 2015, 143, 181103.

(45) Coriani, S.; Koch, H. Erratum: "Communication: X-ray absorption spectra and core-ionization potentials within a corevalence separated coupled cluster framework" [J. Chem. Phys. 143, 181103 (2015)]. J. Chem. Phys. 2016, 145, 149901.

(46) Langhoff, P. W. Stieltjes imaging of atomic and molecular photoabsorption profiles. Chem. Phys. Lett. 1973, 22, 60-64.

(47) Müller-Plathe, F.; Diercksen, G. H. Electronic Structure of Atoms, Molecules and Solids. Proceeding of the II Escola Brasileira de Estructure Eletronica, Olinda, Brazil, July 17-22, 1989; Canuto, S., D'Albuquerque e Castro, J., Paixao, F. J., Eds.; World Scientific, Olinda, Brazil, 1990; pp 1-29.

(48) Lin, N.; Santoro, F.; Zhao, X.; Toro, C.; De Boni, L.; Hernández, F. E.; Rizzo, A. Computational Challenges in Simulating and Analyzing Experimental Linear and Nonlinear Circular Dichroism Spectra. R-(+)-1,1'-Bis(2-naphthol) as a Prototype Case. J. Phys. Chem. B 2011, 115, 811-824.

(49) Casida, M. E.; Jamorski, C.; Casida, K. C.; Salahub, D. R. Molecular excitation energies to high-lying bound states from timedependent density-functional response theory: Characterization and correction of the time-dependent local density approximation ionization threshold. J. Chem. Phys. 1998, 108, 4439-4449.

(50) Lehtonen, O.; Sundholm, D.; Send, R.; Johansson, M. P. Coupled-cluster and density functional theory studies of the electronic excitation spectra of trans-1,3-butadiene and trans-2-propeniminium. J. Chem. Phys. 2009, 131, 024301.

(51) Epifanovsky, E.; Kowalski, K.; Fan, P.-D.; Valiev, M.; Matsika, S.; Krylov, A. I. On the electronically excited states of uracil. J. Phys. Chem. A 2008, 112, 9983-9992.

(52) Schreiber, M.; Silva-Junior, M. R.; Sauer, S. P. A.; Thiel, W. Benchmarks for electronically excited states: CASPT2, CC2, CCSD, and CC3. J. Chem. Phys. 2008, 128, 134110.

(53) Clark, L. B.; Peschel, G. G.; Tinoco, I. Vapor Spectra and Heats of Vaporization of Some Purine and Pyrimidine Bases. J. Phys. Chem. 1965, 69, 3615-3618.

(54) Avila Ferrer, F. J.; Santoro, F.; Improta, R. The excited state behavior of cytosine in the gas phase: A TD-DFT study. Comput. Theor. Chem. 2014, 1040-1041, 186-194. 
(55) Liu, Y.; Martínez-Fernández, L.; Cerezo, J.; Prampolini, G.; Improta, R.; Santoro, F. Multistate coupled quantum dynamics of photoexcited cytosine in gas-phase: Nonadiabatic absorption spectrum and ultrafast internal conversions. Chem. Phys. 2018, 515, $452-463$.

(56) Avila Ferrer, F. J.; Cerezo, J.; Stendardo, E.; Improta, R.; Santoro, F. Insights for an Accurate Comparison of Computational Data to Experimental Absorption and Emission Spectra: Beyond the Vertical Transition Approximation. J. Chem. Theory Comput. 2013, 9, 2072-2082.

(57) Improta, R.; Lami, A.; Barone, V.; Santoro, F. Time-dependent and time-independent approaches for the computation of absorption spectra of Uracil derivatives in solution. Int. J. Quantum Chem. 2010, 110, 624-636.

(58) Barbatti, M.; Aquino, A. J. A.; Szymczak, J. J.; Nachtigallova, D.; Hobza, P.; Lischka, H. Relaxation mechanisms of UV-photoexcited DNA and RNA nucleobases. Proc. Natl. Acad. Sci. U.S.A. 2010, 107, 21453-21458.

(59) Hettema, H.; Jensen, H. J. Aa.; Jørgensen, P.; Olsen, J. Quadratic response functions for a multiconfigurational self-consistent field wave function. J. Chem. Phys. 1992, 97, 1174-1190.

(60) Sałek, P.; Vahtras, O.; Helgaker, T.; Ågren, H. Densityfunctional theory of linear and nonlinear time-dependent molecular properties. J. Chem. Phys. 2002, 117, 9630-9645.

(61) Crespo-Hernández, C. E.; Cohen, B.; Hare, P. M.; Kohler, B. Ultrafast excited-state dynamics in nucleic acids. Chem. Rev. 2004, 104, 1977-2020.

(62) Santoro, F.; Barone, V.; Gustavsson, T.; Improta, R. Solvent effect on the singlet excited-state lifetimes of nucleic acid bases: A computational study of 5-fluorouracil and uracil in acetonitrile and water. J. Am. Chem. Soc. 2006, 128, 16312-16322.

(63) Gustavsson, T.; Bányász, Á.; Lazzarotto, E.; Markovitsi, D.; Scalmani, G.; Frisch, M. J.; Barone, V.; Improta, R. Singlet ExcitedState Behavior of Uracil and Thymine in Aqueous Solution: A Combined Experimental and Computational Study of 11 Uracil Derivatives. J. Am. Chem. Soc. 2006, 128, 607-619.

(64) Pecourt, J.-M. L.; Peon, J.; Kohler, B. DNA Excited-State Dynamics: Ultrafast Internal Conversion and Vibrational Cooling in a Series of Nucleosides. J. Am. Chem. Soc. 2001, 123, 10370-10378.

(65) Hare, P. M.; Crespo-Hernandez, C. E.; Kohler, B. Internal conversion to the electronic ground state occurs via two distinct pathways for pyrimidine bases in aqueous solution. Proc. Natl. Acad. Sci. U.S.A. 2007, 104, 435-440.

(66) Oliveira, E. F.; Shi, J.; Lavarda, F. C.; Lüer, L.; Milián-Medina, B.; Gierschner, J. Excited state absorption spectra of dissolved and aggregated distyrylbenzene: A TD-DFT state and vibronic analysis. J. Chem. Phys. 2017, 147, 034903.

(67) Avila Ferrer, F. J.; Santoro, F. Comparison of vertical and adiabatic harmonic approaches for the calculation of the vibrational structure of electronic spectra. Phys. Chem. Chem. Phys. 2012, 14, 13549-13563.

(68) Picconi, D.; Lami, A.; Santoro, F. Hierarchical transformation of Hamiltonians with linear and quadratic couplings for nonadiabatic quantum dynamics: Application to the $\pi \pi^{*} / \mathrm{n} \pi^{*}$ internal conversion in thymine. J. Chem. Phys. 2012, 136, 244104.

(69) Improta, R.; Barone, V.; Lami, A.; Santoro, F. Quantum Dynamics of the Ultrafast $\pi \pi^{*} / \mathrm{n} \pi^{*}$ Population Transfer in Uracil and 5-Fluoro-Uracil in Water and Acetonitrile. J. Phys. Chem. B 2009, 113, 14491-14503.

(70) Dreuw, A.; Wormit, M. The algebraic diagrammatic construction scheme for the polarization propagator for the calculation of excited states. Wiley Interdiscip. Rev.: Comput. Mol. Sci. 2015, 5, 82-95.

(71) Silva-Junior, M. R.; Schreiber, M.; Sauer, S. P. A.; Thiel, W. Benchmarks for electronically excited states: time-dependent density functional theory and density functional theory based multireference configuration interaction. J. Chem. Phys. 2008, 129, 104103.
(72) Starcke, J. H.; Wormit, M.; Schirmer, J.; Dreuw, A. How much double excitation character do the lowest excited states of linear polyenes have? Chem. Phys. 2006, 329, 39-49. 